\title{
WZ35 Induces Metabolic Reprogramming of Hepatoma Cells Through ROS-Mediated Inhibition of YAP-GLUT1 Axis
}

\section{Lihua Wang}

Wenzhou Medical University

Zheng Zhu

Wenzhou Medical University

Qi Liang

Wenzhou Medical University

Yecheng Tao

Wenzhou Medical University

Junbo Chen

Wenzhou Medical University

Gaowei Jin

Wenzhou Medical University

Yaoyao Zhong

Wenzhou Medical University

Jichen Dai

Wenzhou Medical University

Ruixia Dai

Wenzhou Medical University

Zhixiang Wang

Wenzhou Medical University

Lingjie Zhou

Wenzhou Medical University

Shouyu Ke

Wenzhou Medical University

Xiaokun Lin

Wenzhou Medical University

\section{Linhua Lan}

Wenzhou Medical University First Affiliated Hospital: The First Affiliated Hospital of Wenzhou Medical University

\section{Tongke Chen ( $\nabla$ dry136@163.com )}

Wenzhou medical university https://orcid.org/0000-0002-5485-2431 
Research

Keywords: hepatocellular carcinoma, WZ35, YAP, GLUT1, metabolic reprogramming

Posted Date: September 21st, 2021

DOl: https://doi.org/10.21203/rs.3.rs-898527/v1

License: (c) (i) This work is licensed under a Creative Commons Attribution 4.0 International License. Read Full License 


\section{Abstract}

Background: The significant changes in a series of biochemical activities during the process of tumorigenesis and development, including those in glucose, lipid, and amino acid metabolism can contribute to the ability of the cancer cells to proliferate indefinitely. WZ35 is a new small molecule YAP inhibitor, which can mediate YAP activity to inhibit the growth of gastric cancer, breast cancer and hepatocellular carcinoma. In this article, we explored how WZ35 can modulate YAP activity to influence the metabolism of hepatocellular carcinoma (HCC) cells to inhibit their proliferation activity.

Methods: The Gene Expression Omnibus (GEO) data, the Cancer Genome Atlas (TCGA) data, immunohistochemistry $(\mathrm{IHC})$ and preclinical mouse model was utilized to detect the differential expression and vital role of YAP in HCC cells. A series of in vitro and in vivo experiments were performed to explore the antitumor activity of WZ35 and how it can target YAP activity to inhibit the tumor progression. UCSC combined JASPAR public databases were used to predicted possible binding sites of TEAD on GLUT1 promoter. Additionally, seahorse energy metabolism experiments and metabolomics analysis were utilized to explore the possible metabolic changes induced by WZ35. The clinical use of YAP and GLUT1 was verified by bioformatics and tissue microarrays of immunocytochemistry.

Results: WZ35 can significantly attenuate the proliferation and growth of HCC. In terms of mechanism(s), it was demonstrated that the suppressive effects of WZ35 on YAP were achieved by promoting ROS production and the drug can exert its significant YAP inhibitory capacity to decrease the level of GLUT1, a glucose transporter located on the surface of cytomembrane, thereby resulting in a significant decrease in the ability of cells to take up glucose and thus perturb the metabolism. Moreover, through bioinformatics mining, it was proposed that YAP/GLUT1 has the potential to serve as promising target for HCC therapy.

Conclusions: Our findings indicate that potential inhibitory effects of WZ35 were achieved by affecting the ROS-YAP-GLUT1 signal axis to induce the metabolic reprogramming of hepatocellular carcinoma cells. YAP/GLUT1 could serve as an important molecular target for both the diagnosis and treatment of HCC.

\section{Background}

Hepatocellular carcinoma (HCC), as the fourth most common malignant disease in the world, has become one of the important public health problems [1]. The mechanisms underlying the occurrence and development of hepatocellular carcinoma are still unclear. One of the most prominent characteristic of the tumor cells is that of uncontrolled rapid proliferation, so it needs to balance catabolism and synthetic metabolism, such as reprogramming in glucose, amino acid, lipid metabolism and so on, to achieve a delicate balance between reduction equivalent supply and synthetic biomacromolecules, which is called metabolic reprogramming of tumor cells [2]. Based on this phenomenon, there are several studies related to the tumor therapy that can target tumor metabolism [3]. A number of reports have found that the low glucose microenvironment caused by large consumption of glucose in tumor cells can limit the 
production of T cells, resulting in reduced mTOR activity, glycolysis ability and IFN-gamma production and thereby facilitating tumor progression [4]. At the same time, other studies have found that ketogenic diet or exogenous supplements can induce ketosis, significantly reduce blood glucose and insulin, increase ketone bodies, modulate the acidic tumor microenvironment, as well as slow down the tumor progression and potential symptom burden [5]. Ketosis is also being explored as a potential anti-cancer treatment [6].

Glucose, as the most important energy source of the cell, plays an extremely important role in the progression of the tumor cells [7]. The results of prior studies have shown that [8] the blood glucose level could be directly and positively correlated with the tumor growth. 'Warburg effect ' refers to an important change in the glucose metabolism of tumor cells [9]. Glycolysis of tumor cells is still active even under aerobic conditions, showing high glucose uptake rate, high lactic acid content of the metabolites, and high ratio of glycolysis of the cancer cells to meet the various metabolic needs of the cells. High glucose uptake requires a large amount of the glucose transport from the tissue fluid into the cell, which is mainly mediated through the different glucose transporters (GLUTs). At present, there are 14 members belonging to this family, among which GLUT1 is the most important [10]. GLUT1 is one of the main members of the glucose transporter family. It consists of 12 transmembrane helixes, which constitute two N-terminal and C-terminal domains. These two domains constitute a typical inward conformation that can assist the folding of the transporter superfamily [11]. As a rate-limiting factor in glucose transport process, glucose is mainly transported into the cells. Since tumor cells need to express a great quantity of GLUT1 to meet their huge energy needs, GLUT1 can essentially serve as a potential tumor cell marker [12]. A number of studies have found that GLUT1 expression in a variety of tumors is increased, such as that of lung cancer, hepatocellular carcinoma, gastric cancer, breast cancer, ovarian cancer, and colorectal cancer [13-16]. At the same time, Wang et al demonstrated that both GLUT1 antibody and shRNA significantly inhibited the proliferation and increased the chemosensitivity of tumor cells by disrupting the glucose transport activity of GLUT1 and reducing the expression of GLUT1 [17]. Kurahara et al found that the prognosis of patients with pancreatic ductal adenocarcinoma was directly related to the expression of GLUT1 [18]. Because of the importance of GLUT1 in the regulation of energy metabolism of tumor cells, drug targeting GLUT1 to regulate the glucose transport could be of great significance in cancer therapy.

YAP, also know as YAP1, is a transcription regulator of the Hippo pathway and has many important biological properies in cell development, tissue homeostasis and cancer. The Hippo pathway is a very conserved pathway that can regulate genes transcription. When the pathway is not activated, the active LATSs induces the phosphorylation of YAP, which caused it to stagged in cytoplasm [19]. But when the LATSs is not active, YAP accumulates in the nucleus, binding to the TEAD, a transcription factor, and promotes the expression of target genes. Therefore, YAP is located in the core part of the cell signaling network and plays a vital role in organ growth, cell proliferation, stem cell amplification, and cell fate decision [20]. For example, overexpression of YAP in the liver of transgenic animals can induce the proliferation of mature hepatocytes and ultimately lead to a four-fold increase in liver mass [21]. Its overexpression can also cause hyperproliferation of and depressed differentiation of neural progenitor cells [22]. Many other organs can be effected by YAP expression, too. It is worth mentioning that YAP has 
become the core determinant of human cancer malignancies [23]. It has been detected in late-stage ovarian, colon, gastric, liver, esophageal, non-small-cell lung cancers and lobular type of invasive breast cancers to foster their proliferation, progression and migration. In recent years, studies have found that YAP is an emerging node that coordinates cell nutrition and tissue homeostasis, and participates in the regulation of cell metabolism such as glycolysis, lipogenesis, and glutaminolysis [20]. Since metabolism is also closely related to cell proliferation, what is the relationship between YAP's ability to regulate cell proliferation and metabolic changes?

WZ35 is a small molecule YAP inhibitor synthesized by our team [24]. Our team has confirmed that the curcumin derivative WZ35 can significantly affect the growth of gastric cancer [25], hepatocellular carcinoma [26] and breast cancer [27] by targeting YAP. In this article, we further found that WZ35 could negatively regulate the metabolism of HCC cells mediated through GLUT1. However, the relationship between YAP and GLUT1 remains unclear. In an elegant study, Andrew G Cox et al used zebrafish embryos as a model to prove that YAP regulates the expression of GLUT1 and thus induces the regeneration of organs [28]. But the effect on the metabolism and regulation mechanism remains to be further explored.

In this report, we found a novel YAP inhibitor WZ35 that could inhibit the intake of glucose through ROSYAP-GLUT1 axis. We identified new function of YAP and GLUT1 that were important for the quality of prognosis of HCC patients and might provide new insight into the potential mechanism of the pathogenesis of hepatocellular carcinoma. Further experiments demonstrated glucose intake inhibition inducing metabolic reprogramming and thereby suppressing the proliferation of HCC cells.

\section{Materials And Methods}

\section{Reagents}

Curcumin was obtained from Sigma (MO, USA). WZ35, a monocarbonyl curcumin analog, was prepared as described in prior studies [29]. Cell Counting Kit-8 (CCK-8) and horseradish peroxidase (HRP)conjugated anti-rabbit and anti-mouse IgG and N-acetyl-I-cysteine (NAC) were purchased from Beyotime (Haimen, China). Cell Apoptosis DAPI Detection Kit, DCFH-DA ROS detection kit, and pEGFP-hYAP 1 were obtained from Addgene (Shanghai, China). A Hippo Signaling Antibody Sampler Kit (\#8579), antiPhospho-SAPK/JNKThr183/Tyr185 (\#4668), anti-Cleaved caspase 3 (\#9661), anti-Bax (\#2774), anti-Bcl-2 (\#2870), anti-E-cadherin (\#8834S), anti-GLUT1 (\#73015), anti-N-cadherin (\#13116S), and anti-GAPDH (\#D16H11) were purchased from Cell Signaling Technology (USA).

\section{Cell culture}

The human HCC cell lines including HCCLM3, HepG2 and Huh7 were obtained from the Institute of Biochemistry and Cell Biology, Chinese Academy of Sciences. The cells were grown using Dulbecco's Modified Eagle's Medium (DMEM) containing 10\% FBS (Life Technologies) and penicillin/streptomycin at $37^{\circ} \mathrm{C}$ in a humidified $5 \% \mathrm{CO}_{2}$ incubator. 


\section{Patient samples}

In total, 110 matched HCC tumor samples and precancerous control tissues were isolated from untreated patients undergoing surgical tumor resection at the Department of Hepatopancreatobiliary Surgery of the First Affiliated Hospital of Wenzhou Medical University, Wenzhou, China, between 2010 and 2014. The tumor samples were separated into two different portions, with one portion being used for routine histopathological examinations, and the rest being snap-frozen and stored at $-80^{\circ} \mathrm{C}$ for future studies. $\mathrm{HCC}$ was pathologically confirmed in all patients, with tumors staging being determined based upon the AJCC/UICC (Union for International Cancer Control/American Joint Committee on Cancer) and WHO classification. The Board and Ethical Committee of the First Affiliated Hospital of Wenzhou Medical

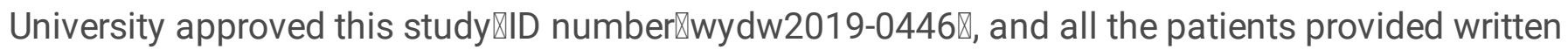
informed consent.

\section{Bioinformatics analysis}

The GENE EXPRESSION OMNIBUS (GEO) database was searched using the terms "gene expression", "hepatocellular carcinoma", and "Homo sapiens". We selected the GSE14520 dataset as it met the quality control and the sample size criteria. The R software (version.4.0.3) affyPLM package was used for the regression analyses and normalization. Based on quality filtering, two healthy control microarray samples were excluded, while the remaining 41 microarrays (22 HCC samples, 19 adjacent control samples) based on the GPL571 platform (Affymetrix Human Genome U133A 2.0 Array) were retained for analysis. The RStudio Limma package was used to identify the differentially expressed genes $(P<0.05$ and | logFC (Fold Change) $\mid \geq 1$ ), after which probe names were converted to gene names. The heatmap.2 package was used to visualize differences in gene expression between the samples, with appropriate functions also being used to draw the boxplots, survival curves, and volcano plots in $\mathrm{R}$ software (version.4.0.3).We used database for annotation, visualization and integrated discovery for $\mathrm{GO}$ enrichment analysis. We also performed pathway enrichment analysis using pathway data obtained from the FTP service of KEGG (Kyoto Encyclopedia of Genes and Genomes). JASPAR [30] and USCS [31] were used to predicted binding sites, conservation and histone modification.

\section{Cell proliferation assay}

A CCK-8 kit was used to measure the cellular proliferation. Briefly, HCC cells were plated in 96-well plates $\left(8 \times 10^{3} /\right.$ well) overnight, after which they were untreated or treated with curcumin $(10 \mu \mathrm{g} / \mathrm{mL}), \mathrm{WZ35}(10$ $\mu \mathrm{g} / \mathrm{mL}), \mathrm{NAC}(5 \mathrm{mM})$. For some experiments, a range of drug concentrations $(0,5,10,20$, and $40 \mu \mathrm{g} / \mathrm{mL})$ were used instead. WZ35 and curcumin were prepared using $0.03 \%$ DMSO; NAC was prepared in PBS and diluted using complete media to appropriate concentrations for use. After drug treatment, the CCK-8 kit was used to measure the viability with absorbance at $450 \mathrm{~nm}$ being quantified via microplate reader (Spectramax m5, Molecular Devices).

\section{Colony formation assay}


Initially, HCCLM3 cells were plated in 6-well plates (5,000/well) with curcumin ( $1 \mu \mathrm{g} / \mathrm{mL})$, WZ35 (1 $\mu \mathrm{g} / \mathrm{mL})$, and/or NAC ( $5 \mathrm{mM}$ ). The plate incubated until individual colonies were formed containing approximately 50 cells. The cells were then washed in PBS, fixed for 20 minutes with methanol, stained for 15 minutes with crystal violet, washed with distilled water, and colonies were counted using the ImageJ software.

\section{DAPI staining for cells}

Apoptosis was measured by plating HCCLM3 cells in 6-well plates overnight ( $5 \times 10^{5} /$ well). The cells were then treated using WZ35 or curcumin (both $10 \mu \mathrm{g} / \mathrm{mL}$ ) for $18 \mathrm{~h}$, after which they were washed using PBS, fixed for 15 minutes in 4\% paraformaldehyde (PFA), and stained for 15 minutes with 4',6-diamidino-2phenylindole (DAPI) $(400 \mu \mathrm{L})$. The cells were thereafter imaged via microscope (NIKON, Japan) with 100 magnification.

\section{Western blotting}

Radio immunoprecipitation assay (RIPA) lysis buffer obtained from Beyotime (Haimen, China) was used to isolate the proteins from the cells or to homogenize the tumor tissues. The samples were then spun for 15 minutes at $12,000 \mathrm{rpm}$, and equal amounts of supernatant protein ( $10 \mu \mathrm{g} / \mathrm{sample})$ were separated via sodium dodecyl sulfate-polyacrylamide gel electrophoresis (SDS-PAGE) $(8,10$ or $12 \%)$ prior to transfer onto a poly (1,1-difluoroethylene) (PVDF) membrane (Millipore, Burlington, MA, USA). The blots were blocked for 90 minutes using 5\% skim milk in TRIS hydrochloride \& Tween (TBST), followed by an overnight incubation with the various primary antibodies at $4{ }^{\circ} \mathrm{C}$. After washing three times in TBST (10 minutes/wash), the blots were incubated for $1 \mathrm{~h}$ with horseradish peroxidase (HRP)-linked secondary antibodies (1:2000), after which they were washed again and protein bands were detected using an electrochemiluminescence $(E C L)$ reagent (Thermo Scientific, IL, USA).

\section{Transmission Electron Microscopy (TEM)}

Curcumin- and WZ35-induced changes in the cellular structure were visualized via TEM, with DMSO being used as a control. Initially, the treated cells were pre-fixed overnight in $2 \%$ glutaraldehyde, followed by a 2 h post-fix in $1 \%$ osmium tetroxide. Ethanol and acetone gradients were then used to dehydrate the cells, after which the cell pellets were embedded in ethanol and acetone. Ultrathin pellet sections were then stained using $2 \%$ aqueous uranyl acetate and lead citrate, after which a TEM instrument (Wenzhou Medical University, H-7500, HITACHI, Japan) was used to image the cells with 26500 magnification.

\section{Oxidative phosphorylation and glycolysis measurements}

A Seahorse XF96 Extracellular Flux Analyzer (Agilent Technologies, CA, USA) was used for oxygen consumption rate (OCR) and extracellular acidification rate (ECAR) measurements. Briefly, curcumin or WZ35 doses were used to treat HCCLM3 cells for $6 \mathrm{~h}$, after which $2 \times 10^{4}$ cells were added to machinespecific plates. Following probe calibration, OCR was measured via sequentially injecting oligomycin 
(ATP synthase inhibitor; $1 \mu \mathrm{M}$ ), FCCP (uncoupler; $0.5 \mu \mathrm{M}$ ), rotenone (complex I inhibitor; $1 \mu \mathrm{M}$ ), and antimycin A (complex III inhibitor; $2 \mu \mathrm{M}$ ). ECAR was measured using the cells treated in the same manner via injection of glucose $(10 \mathrm{mM})$, oligomycin $(1 \mu \mathrm{M})$, or 2-DG $(100 \mathrm{mM})$. In addition, the basal cellular glycolytic rate of untreated, curcumin-treated, and WZ35-treated HCCLM3 was calculated.

\section{Flow cytometry analysis for ROS determination}

ROS production was quantified by staining the cells for 30 minutes with $10 \mu \mathrm{M}$ 2,7-dichlorofluo-rescein diacetate (DCFH-DA) in DMEM at $37^{\circ} \mathrm{C}$. The cells were then treated for $2 \mathrm{~h}$ with corresponding compounds $(10 \mu \mathrm{g} / \mathrm{mL})$, washed twice with DMEM, and DCFH-DA fluorescence was quantified by BD Accuri TM C6 flow cytometer (BD, Franklin Lakes, NJ).

\section{Detection of glucose in the supernatant}

HCCLM3 cells were plated in 3 dishes $(60 \mathrm{~mm})$ with DMSO $₫ 10 \mu \mathrm{L} / \mathrm{mL} \varangle \mathrm{WZZ35}(10 \mu \mathrm{g} / \mathrm{mL})$ and blank. After a $18 \mathrm{~h}$ drug treatment, the supernatant was collected and analyzed. According to Beckman Kurt biochemical analysis system, glucose determination kit (Beckman Coulter, CA, USA) was used to detect the glucose content in the culture medium supernatant. In the presence of adenosine triphosphate (ATP) and magnesium ions, the glucose was phosphorylated by hexokinase (HK) to produce glucose-6phosphate and adenosine diphosphate (ADP). Glucose-6-phosphate dehydrogenase (G6P-DH) specifically can oxidize glucose-6-phosphate to 6-phosphogluconic acid. Oxidized nicotinamide adenine dinucleotide $\left(\mathrm{NAD}^{+}\right)$was also reduced to nicotinamide adenine dinucleotide (NADH). The increase of absorbance at $340 \mathrm{~nm}$ was proportional to the glucose concentration in the sample.

\section{Glucose metabolism test}

A CCK-8 kit was used to measure the cellular proliferation. HCCLM3 cells were plated in 96-well plates ( $8 \times 10^{3} /$ well) overnight, medium was changed to sugar-free medium the next day, after which the cells were left untreated or treated with Glucose (1 mM), Glucose $(25 \mathrm{mM}), 2-D G(1 \mathrm{mM})$, WZ35 $(10 \mu \mathrm{g} / \mathrm{mL})$. After an 18h-drug treatment, the CCK-8 kit was used to measure the viability, with absorbance at $450 \mathrm{~nm}$ being quantified via microplate reader (Spectramax m5, Molecular Devices).

\section{Metabolomics analysis}

TCA: Targeted metabolite analysis was performed on a Xevo G2-XS QT of mass spectrometry coupled with an ACQUITY UPLC (Ultra Performance Liquid Chromatography) system and data analysis was done with Progenesis QI (all from Waters, Milford, MA, USA). The metabolites in a homogenate of $1 \times 10^{6}$ cells were extracted using chloroform, methanol, and water at a ratio of 8:4:3 and resuspended in $1 \rrbracket$ acetonitrile. For UPLC, the samples were injected on an HSS T3 column $(100 \times 2.1 \mathrm{~mm}, 1.8 \mu \mathrm{m})$ using an 8-min gradient containing flow phase $A(0.1 \otimes$ formic acid-water) and flow phase $B(0.1 \%$ formic acidacetonitrile) at a flow rate of $0.5 \mathrm{ml} / \mathrm{min}$. For the negative ion mode, the capillary energy and sample cone were set as $2000 \mathrm{~V}$ and $20 \mathrm{~V}$, respectively. Scan time was set at $0.1 \mathrm{~s}$ intervals for $60 \mathrm{~s}$. 
Other metabolomics: A. 95\% water: $5 \%$ acetonitrile $+10 \mathrm{mM}$ ammonium acetate $+\mathrm{NH}_{4} \mathrm{OH}(\mathrm{PH}=10)$; $\mathrm{B}$. $95 \%$ acetonitrile : $5 \%$ water $+10 \mathrm{mM}$ ammonium acetate $+\mathrm{NH}_{4} \mathrm{OH}(\mathrm{PH}=10)$. Waters Acquity UPLC BEH Amide $2.1 \times 100 \mathrm{~mm}, 1.7 \mu \mathrm{m}$. Time, flow velocity $\varangle \mathrm{mL} / \mathrm{min} \rrbracket, A 1 \%, B 1 \%$ were arranged in the following order: initial, 0.4, 1, 99; 0.1, 0.4, 1, 99; 6, 0.4, 70, 30; 6.5, 0.4, 1, 99; 10, 0.4, 1, 99.

\section{Tumor transplantation}

Female BALB/c nude mice (6-8 weeks old; $20 \mathrm{~g}$ ) from Wenzhou Medical University Laboratory Animal Center were housed under SPF conditions. These animals were subcutaneously injected with a $100 \mu \mathrm{L}$ volume containing $5 \times 10^{6}$ HCCLM3 cells. When tumors grew to $100 \mathrm{~mm}^{3}$, the mice were randomized into 4 groups ( $n=6 /$ group) that were intraperitoneally administered saline (Mock) vehicle, curcumin ( 25 $\mathrm{mg} / \mathrm{kg}$ ), or WZ35 (25 mg/kg) daily for 17 consecutive days. Animal body weight and tumor volumes were monitored every other day, with the latter being calculated as follows: tumor volume $=$ length $\times$ width $\times$ height/2. After 17 days, sodium pentobarbital was used to euthanize mice, and the tumor tissues were collected to make paraffin sections for HE staining. Our project was conducted under guidelines approved by the Experimental Animal Ethics Committee of Wenzhou Medical University $\mathbb{\text { ID }}$ number $\rrbracket w y d w 2019$ 0446冈.

\section{Tissue microarrays (TMAs) and IHC staining}

HCC TMAs composed of 110 FFPE tissue sections ( $4 \mu \mathrm{m}$ thick) were prepared the same way as previously described by Guttà $\mathrm{C}$ et al [32]. TMA IHC staining was conducted based on a standard approach. Briefly, the sections were deparaffinized using xylene, rehydrated with an ethanol gradient, treated to quench peroxidase activity, blocked with $5 \%$ normal goat serum, and incubated with antiYAP/GLUT1 overnight at $4{ }^{\circ} \mathrm{C}$. As a negative control, the antibody was omitted. The slides were then probed with biotin-labeled goat anti-rabbit IgG, treated with a streptavidin peroxidase solution (SABC kit, Boster, Wuhan, China), and then 3,3-diaminobenzidine (Boster, Wuhan, China) in PBS with $0.05 \% \mathrm{H}_{2} \mathrm{O}_{2}$ was used to treat the samples for $5 \mathrm{~min}$ at the room temperature for color development, after which the samples were counterstained using hematoxylin.

\section{IHC staining assessment}

Two pathologists blinded to the samples evaluated the clinicopathological information independently and scored IHC images of TMA samples. We utilized the Image-Pro Plus 6.0 (IPP) (Media Cybernetics, $M D, U S A)$ to calculate the integrated optical density (IOD) for the stained images, with this value corresponding to total staining per sample area [33]. Entire TMAs were scanned in order to quantify protein levels in the TMA levels. IHC staining was evaluated based on immunoreactive score (IRS) values as described in the prior studies [26], with these scores reflecting both staining intensity and the percentage of the positive cells. The percent positivity was stained as follows: $1(\leq 10 \%), 2(10-50 \%), 3$ $(50-80 \%)$, or $4(\geq 80 \%)$. The staining intensity was scored as: 0 (negative), 1 (weak), 2 (moderate), or 3 (strong). These two scores were then multiplied together to yield the IRS value $(0-12)$, with these values being deemed either low $(\leq 6)$ or high $(>6)$. 


\section{Statistical analysis}

SPSS 19.0 (SPSS Inc., IL, USA) was utilized for statistical analysis. The data has been represented as the means \pm SD from three independent experiments, and was compared via Student's t-tests. $P<0.05$ was set as the significance threshold.

\section{Results}

\section{YAP upregulation in HCC corresponds to poorer patient outcomes}

$Y A P$, as an oncogenic gene, plays an important part in the occurrence and development of cancer [34-35]. To understand the possible role of $Y A P$ in HCC, we searched the Gene Expression Omnibus (GEO) datasets. YAP1 is regarded the most common subtypes of $Y A P$, which plays a significant role and exhibited the highest content among YAPs. We began by analyzing YAP1 expression levels in the GSE14520 HCC gene expression dataset. The differential expression of YAP1 was visualized in the volcano map and Boxplots (Fig. 1a,b), revealing the upregulation of $Y A P 1$ in HCC (log FC $>2, P<<0.001)$. Heat map similarly supported this HCC-specific upregulation of YAP1 (Fig. S1a) $(P<<0.05)$. The visualization of enrichment analyses of DEGs have been shown in Supplementary Fig. S1b.

In order to confirm these bioinformatics findings, we next extracted fresh protein from the tissue samples of $18 \mathrm{HCC}$ patients and corresponding adjacent controls. The results of Western blotting confirmed that YAP expression was significantly higher in the tumor samples relative to control samples (Fig. 1c), which was consistent with our in silico findings. The results of IHC staining of YAP in TMAs composed of 110 patient-derived HCC samples was analyzed to assess the relationship between YAP expression and the different clinicopathological findings of HCC patients. Apparently, YAP expression was highly significant and a positive association with tumor histological TNM stage $(P<0.001)$ was noted (Fig. 1d,e). Moreover, the expression of YAP in well-differentiated (G1) HCC tissues was significantly lower as compared with the tissues that were moderately (G2) $(P=0.003)$ and poorly ( G3 $)(P<0.001)$ differentiated, and the levels of YAP were significantly higher in G3 samples in contrast to G2 samples $(P<0.001)$ (Fig. S1C). These findings indicated that an upregulation of YAP can coincide with the progression of HCC. We next examined the relationship between levels of YAP protein and overall survival (OS) of HCC patients via Kaplan-Meier analyses, and found that the high expression of YAP was closely related to poorer OS, while low related to better. (Fig. 1f) $(P<0.01)$. We then conducted univariate and multivariate analyses to identify the possible relationship between the expression of YAP and clinicopathological features of other HCC patients (Table1). In univariate analyses, the levels of YAP, tumor nodules, and venous invasion were all found to serve as significant prognostic factors, while in multivariate analyses, the expression of YAP was found to be independently associated with the prognoses of HCC patients. Together, these results indicated that YAP protein was overexpressed in HCC and may serve as an important target for therapeutic intervention.

\section{WZ35 inhibited YAP from entering the nucleus and thereby affecting its function}


In order to explore the functional role of YAP in HCC, we first knocked down or over-expressed this protein in HCCLM3 for various assays (Fig. 2a). YAP knockdown significantly reduced the colony formation rates as compared to the control cells, whereas the opposite facilitated this process (Fig. 2b,c). Moreover, consistent results were observed in a CCK-8 assay as well (Fig. 2d). These findings clearly suggested that the proliferation of HCC cells was regulated by YAP.

After shuttling from cytoplam into the nucleus, YAP can regulate gene transcription and ultimately promote proliferation, apoptosis and morphogenesis [36]. We have previously shown that the curcuminderived WZ35 compound (Fig. 2e) can cause a significant downregulation in the protein levels of YAP. To explore the localization of YAP's in HCC cells, we performed immunofluorescence assay in WZ35-treated HCCLM3 cells via confocal microscopy. It was observed that the green signals were significantly diminished following WZ35 treatment, particularly in the nuclei of HCC cells, with DAPI (blue) used for nuclear visualization (Fig. 2f), thereby indicating that WZ35 was able to retard the nuclear translocation of YAP and its subsequent binding with TEA domain (TEAD). Consistent with this finding, WZ35 significantly increased the phosphorylation of YAP at serine 127 residue (Fig. $\mathbf{2 g}$ ). It has been shown that YAP can regulate the transcription primarily via TEAD family transcription factors, thereby affecting cellular proliferation. In a non-phosphorylated state, YAP undergoes nuclear translocation and functions as a potential TEAD transcriptional activator, whereas S127 phosphorylation of YAP results in its interaction with 14-3-3 protein and consequent cytoplasmic sequestration [37]. Overall, not only can WZ35 down-regulate YAP signaling, but can effectively hinder its translocation and ability to promote the viability of HCC cells.

\section{WZ35 showed superior anti-cancer activity under in vitro and in vivo settings}

We next attempted to elucidate the relative inhibitory activities of Curcumin and WZ35 in vitro. In a CCK-8 assay, both the compounds caused a dose-dependent inhibition of the tumor cell proliferation, with WZ35 being clearly superior to curcumin (Fig. 3a). Moreover, similar outcomes were observed in the colony formation assays (Fig. 3b). Next, to identify whether inhibition of viability was also accompanied with apoptosis or cell cycle arrest, flow cytometry analysis was performed. Representative cell images indicated that WZ35 substantially induced G2/M cell cycle arrest (Fig. S2a), thus suppress the growth of HCCLM3 cells, and significantly promoted apoptosis (Fig. S2b). To elucidate whether the apoptosis of HCCLM3 cells was correlated with changes in the morphology of the nuclei, we utilized DAPI staining to visualize the changes, and the results revealed that WZ35 treatment induced nuclear shriveling and abnormal nuclear morphology at higher rates as compared to curcumin (Fig. 3c). The levels of various apoptosis-related protein levels were also assessed via Western blotting, with treated cells exhibiting an upregulated levels of pro-apoptotic Bax and cleaved caspase-3 as well as a decrease in expression of anti-apoptotic Bcl-2 (Fig. 3d). These findings clearly suggested that WZ35 was superior to curcumin in inhibiting HCC cells growth in vitro.

In order to extend our range of findings to in vivo settings, nude mice bearing subcutaneous HCCLM3 tumor xenografts were used. We found that both curcumin and WZ35 treatment did not significantly alter 
murine body weight, thereby suggesting no treatment-associated toxicity during the study period (Fig. 3e). While both the compounds were able to inhibit HCC tumor growth, WZ35 was found to be superior in suppressing this growth over a 17-day study period (Fig. 3e,f). According to the result of tumor histological assessment, it was apparent that WZ35-treated tumors revealed lower cellularity and higher tumor necrosis rates as compared with the vehicle and curcumin treatment (Fig. $\mathbf{3 g}$ ). In conclusion, these results suggest that WZ35 may exhibit preferable in vivo efficacy by markedly suppressing HCC tumor growth as compared to curcumin.

\section{WZ35 inhibits HCC growth in a ROS-dependent manner}

To further ascertain the cellular mechanism related to drug-associated apoptosis, we examined the impact of WZ35 on intracellular morphology in HCCLM3 cells via TEM. As shown, apparent mitochondrial dysregulation, scattering, and swelling were observed in the treated cells (Fig. 4a), clearly suggesting that this compound had substantially disrupted mitochondrial homeostasis. Mitochondria are the major intracellular sources of ROS generation owing to electron leakage from the electron transport chain (ETC). We therefore analyzed ROS levels via flow cytometry in WZ35-treated HCCLM3 cells, revealing that this treatment led to a more than 2-fold increase in intracellular ROS levels (Fig. 4b). At normal levels, ROS play an important role in regulating cellular signaling and proliferation, whereas their excess levels can cause deleterious oxidative damage [38]. Hence, an increased ROS generation observed in WZ35-treated cells was likely to contribute to observed reduction in the cellular proliferation.

Thereafter, we utilized N-acetyl-I-cysteine (NAC), an inhibitor of ROS, to pretreat HCCLM3 cells for $2 \mathrm{~h}$ before WZ35 treatment, after which the viability of these co-treated cells was assessed. NAC pretreatment significantly neutralized WZ35-induced HCCLM3 cells proliferation inhibition (Fig. 4c), and correspondingly facilitated the colony forming abilities of WZ35-treated cells (Fig. 4d) while markedly attenuating their apoptotic death (Fig. 4e). At the molecular level, the impact of NAC pretreatment on WZ35-induced changes in the levels of YAP in HCCLM3 cells was analyzed via Western blotting, and the findings revealed that this antioxidant can significantly counteract changes in the expression of YAP caused by WZ35 (Fig. 4f).

All these results suggested that ROS generation was essential in order for WZ35 to suppress growth and proliferation, with the expression of YAP being closely linked to increased ROS production in treated HCC cells.

\section{WZ35 antitumor activity depends upon inhibition of the YAP protein and allows it to regulate the expression of GLUT1}

As we elucidated the direct link between ROS and YAP above, we next proceeded to identify whether YAP was the target affected by WZ35 in drug-treated HCCLM3 cells and its anti-cancer activity. Firstly, YAP was knocked down and over-expressed in HCCLM3 cells. The results of various assays indicated that WZ35 and downregulation of YAP caused a concurrent inhibitory effect on cell viability (Fig. 5c) and clonogenicity (Fig. 5b, Fig. S3b), therefore facilitating their death (Fig. 5a, Fig. S3a), whereas WZ35 with 
co-treatment of upregulation of YAP counteracted the impact of WZ35 on HCCLM3 cells Moreover, an upregulation or downregulation of YAP only was positively or negatively correlated with proliferation and colony forming abilities of HCCLM3 cells.

To further explore the role of YAP in anti-cancer activity, data was downloaded from TCGA database and divided into two groups according to the level of YAP1 expression. We identified differential genes between the two groups by limma R package. Enrichment analysis of these genes based on Gene Ontology (GO) annotation system was conducted. The GO annotation system uses a controlled and hierarchical vocabulary to assign function to genes or gene products in any organism. As displayed in Fig. S3c: the genes corelated with YAP1 expression had enriched GO terms related to energy metabolism especially ATP function. According to pathway information (Fig. S3d) which is pivotal in definiting YAP function, the corelated genes were mainly enriched in many known oncogenic pathway for HCC. Among them, we noticed that the expression of $Y A P 1$ was possitively associated with central carbon metabolism. In the traditional sense, central carbon metabolism, including embden meyerhof pathway (EMP), pentose phosphate pathway (PPP) and tricarboxylic acid cycle (TCA) pathway, is the main source of energy required by organisms. As shown in Fig. 5d,e and Fig. S3j, high expression of YAP1 was significantly associated with central carbon metabolism genes such as SLC2A1, PFKP, G6PD, etc. And SLC2A1 had the most significant correlation. SLC2A1 is the official name of GLUT1 in NCBI. GLUT1 is the main glucose transporter in hepatoma carcinoma cells [9] and plays a vital role in glycolysis. Consistent with above, bioinformatics analysis of GEO database further proved that YAP expression may be closely related to cellular metabolism especially the glycolysis process in hepatoma carcinoma (Fig. S3e,f). GLUT1 was significantly up-regulated in tumor samples versus normal ones (Fig. S3g,h) and high levels of it was associated with a shorter survival in hepatocellular carcinoma patients (Fig. S3i). Western blotting assays unfolded that WZ35 could decrease the expression of YAP as well as GLUT1, and that knockdown of YAP by shRNA enhanced the suppressive effect of WZ35 on GLUT1 (Fig. 5f), whereas overexpression of YAP neutralized it (Fig. S3k). As per the JASPAR [30] database 5 potential TEAD binding sites were present on the GLUT1 promoter (Fig. 5g). UCSC Genome Browser revealed that GLUT1 promoter is highly conserved among mammals and an increased level of histone methylation correlated with H3K4Me3 at the promoter, which indicated active transcription (Fig. S4a).

\section{WZ35 might mediate the inhibition of glucose intake to reduce proliferation}

GLUT1 is a transporter of glucose, which is very important for transportation of glucose in HCC cells [10], and glucose is a major nutrient source for the survival and proliferation of cancer cells because it feeds directly into glycolysis and then also into several other metabolic pathways [39-40]. As it has been demonstrated above that WZ35 could inhibit the expression of glucose transporter GLUT1, the metabolism changes in HCCLM3 cells were detected next. Statistically, after action of curcumin or WZ35, both two curves of the total acidification rate moved down, and the descending degree of the latter outweighed that of the former (Fig. 6a), so did the curves of OCR (Fig. 6d). The glycolytic capacity(Fig. 6b), maximal glycolytic capacity (Fig. 6c) and glycolytic reserve were significantly inhibited by curcumin and WZ35. Consistent with the aforementioned results, basal respiration (Fig. 6e), maximal 
respiration (Fig. 6f) and ATP production (Fig. 6g) were significantly restrained as well. We then detected the absorption of glucose was via glucose determination kit (hexokinase method). Corresponding to the above, WZ35 was found to significantly attenuate the ability to absorb glucose from outside the cells obviously (Fig. 6h). Additionally, by means of CCK-8 assay (Fig. 6i), the absorption of glucose was correlated to the proliferative activity of cells. The cells were cultured in different kinds of mediums with different concentrations of glucose with or without the retardant of glycolysis '2-DG'. According to the results, it was found that the hindrance of glucose absorption by WZ35 plays a vital role in reducing the proliferation activity of the HCC cells. When we artificially retarded the glycolysis process, the ability of WZ35 to inhibit cell proliferation was significantly reduced, thereby suggesting that WZ35 can potentially serve as an effective blocker of glucose absorption, which could possibly exert its anti-proliferative effects by hindering the process of cell glycolysis. To explore the subsequent effects of drug-induced glucose intake on energy metabolism, metabolomics was used to detect the levels of the various intracellular metabolites. As displayed in fig. 6j, treatment with WZ35 enhanced the ratio of $\mathrm{NAD}^{+}$to $\mathrm{NADH}$, signifying the blockade of oxidative phosphorylation. The content of purine metabolites, such as adenosine monophosphate, adenosine triphosphate and guanosine diphosphate, etc. were significantly attenuated in WZ35-treated HCCLM3 cells. Since syntheses of nucleotides was limited to support the process of proliferation, the ability of WZ35 to inhibit cellular proliferation can be explained. In summary, WZ35 restricted the uptake of glucose in HCCLM3 cells, thus leading to inhibition of glycolysis and oxidative phosphorylation. Finally, the subsequent disorder in the purine metabolism could significantly affect the proliferation ability of HCCLM3 cells. The general metablosim changes is shown in fig. $\mathbf{6 k}$.

\section{YAP combined GLUT1 may serve as a valuable prognostic and/or diagnostic biomarker in HCC}

We further explored clinical application of YAP and GLUT1 in above tissue chip in 53 HCC samples. Consistent with YAP, the majority of these HCC patients were GLUT1-positive (Fig. 7a,b) $(P=0.0208)$. Boxplot showed that GLUT1 expression was positively correlated with TNM stage (Fig. 7c). An elevated GLUT1 expression was found to be significantly associated with tumor TNM stage, thereby suggesting that GLUT1 upregulation could coincide with HCC progression. Kaplan-Meier survival analysis showed that high level of GLUT1 was associated with a shorter survival in HCC (Fig. 7d) and the areas under curve (AUC) in ROC curve of GLUT1 were 0.7 (at 1 year), 0.77 (at 3 years) and 0.85 (at 5 years) (Fig. 7e). Since biological information mining has found that YAP and GLUT1 might display a good predictive function in predicting the prognosis of HCC patients (Fig. S5a,d), to further substantiate the survival significance of YAP and GLUT1, the correlation between YAP/GLUT1 high expression and clinical features of HCC patients was analyzed in above 53 clinical cases. The expression of YAP was found to be proportional to GLUT1 (Fig. S5e). High integrated expression levels of YAP and GLUT1 were negatively correlated with survival in HCC patients (Fig. 7f). A nomogram was made according to the result of multivariate logistic proportional hazards analysis which considers Stage I and II as the early stage, Stage III and IV as the late stage. Through fixing the points associated with YAP and GLUT1, the risk of later stage for an individual patient could be calculated (Fig. 7g). The total score was 0-200, and YAP and GLUT1 were calculated and merged. A high score indicated a high risk of late stage HCC. A ROC calibration plot 
was made to assess the internal validity for the constructed nomogram (Fig. 7h).The AUC for combination of YAP and GLUT1 was 0.889 , which was higher than GLUT1 alone (Fig. S5f). Considering these data, we can infer the potential link between YAP and GLUT1 and the integration between them may potentially serve as a valuable prognostic biomarker in HCC patients.

\section{Discussion}

HCC is the third deadliest and sixth most prevalent cancer type globally in 2020 [1]. However, the onset of $\mathrm{HCC}$ is insidious, and once discovered, it is mostly diagnosed in an advanced stage. Additionally, coupled with the lack of effective treatments, the mortality rate of hepatocellular carcinoma is relatively high. Therefore, identification of efficient treatment methods and treatment targets for HCC has become an urgent clinical problem [41]. The genesis and development of tumor cells is a complex process, in which significant changes in a series of biochemical processes such as glucose, amino acid, nucleotide and lipid metabolism can impart unlimited proliferation potential on the tumor cells [42]. The discovery of the Warburg effect in the late 1920s not only emphasized the important role of carbohydrate metabolism in the cancer cells [43], but also opened a new era in the study of tumorigenesis and development of novel therapies from the perspective of metabolism. Therefore, in-depth exploration of various regulatory mechanisms from these metabolic perspectives can potentially serve as an important starting point for the development of effective treatment modalities for HCC.

WZ35 is a small molecule YAP inhibitor independently developed by our team. We have already demonstrated that WZ35 could target YAP to suppress the growth of a variety of tumors, including hepatocellular carcinoma, gastric cancer, and breast cancer [24-27]. Among them, we found by accident that WZ35 could inhibit glycolysis [25]. In this article, we further discovered that WZ35 can block glucose absorption, just as the glucose inhibitor 2-DG (Fig. 6i). According to the desperate demanding of cancer cells for glucose, some scholars suggested intermittent fasting might diminish the progression of tumours [44]. Clinical data showed that fasting can help prevent DNA and immune cells damage caused by chemotherapy [45], promote the regeneration of normal tissues. It is reported that combining fasting with other therapies can improve the therapeutic effect, prevent drug resistance and reduce side effects [46]. However, the lack of nutrition caused by fasting makes the overall anti-cancer effect unsatisfactory. For example, the reduction of glutamine due to glucose deficiency can alleviate immune infiltration in the tumor microenvironment [47]. Because some trace elements and essential amino acids cannot be synthesized in the human body, fasting might induce more diseases such as anemia, neurocognitive deficits, ect [48]. But through CCK-8 assay, we demonstrated that the glucose blocking ability of WZ35 is also the main source for its anti-cancer ability (Fig. 6i). In other words, WZ35 has the same anti-cancer effect as intermittent fasting, but it cleverly avoids the serious side effects caused by nutritional deficiency. Combining the results of in vivo experiments and in vitro experiments, we conclude that WZ35 is a superior anti-cancer candidate drug. What's more, due to its glucose blocking ability, it is also expected to be used as a candidate adjuvant drug for diabetes. WZ35 may reduce the damage caused by the high concentration of glucose infiltration in the tissue fluid through reducing the sensitivity of somatic cells to glucose, which is expected to realize that diabetic patients do not need to go on a painful diet, and 
this function remains to be explored. But how WZ35 blocks glucose absorption inhibition? How does the blockade of glucose absorption affect the metabolism of hepatoma carcinoma cells? These are the exact issues we will explain below.

Here, we identified a brand new signal axis, ROS-YAP-GLUT1 axis. WZ35 can restrain the glucose intake of hepatoma carcinoma cells through this axis and inhibit the proliferation of HCC. Through a series of experiments such as DAPI staining and Western blot analysis, we proved that WZ35 can promote ROS production to stimulate the translocation of YAP into the nucleus and reduce the total amount of YAP. YAP is a pivotal protein involved in the Hippo pathway. Many studies have found that YAP can regulate various important physiological processes such as organ regeneration [49], atherosclerosis [50], ect. In our study, we identified a new function of YAP that can affect the expression of GLUT1 in HCC cells. GLUT1 (Glucose transporter 1) is the most highly conserved and widely found glucose transporter in different cancers [10]. Glucose is primarily transported into cells by GLUT1 [12]. This can explain why WZ35 can regulate glucose uptake. Then, we explored the possible mechanism of YAP regulating GLUT1. YAP, as a transcription regulator, can regulate TEAD-directed trascriptional function when it localizes in the nuclear [51]. We found 5 possible binding sites of TEAD in GLUT1 promoter region and four of them were found an increased level of histone modifications associated with H3K4me3. H3K4me3 is possitively associated with gene expression and gathers around TSS [52]. The discovery of increased H3K4me3 further supports the reliability of these binding sites and we guessed the existence of H3K4me3 might also increase the possibility of combination between TEAD and GLUT1 promoter. So we concluded that the mechanism of YAP regulating GLUT1 may be that YAP enters into the nuclear and directs TEAD to bind with GLUT1 promoter and exercises activation function. As bioinformatics analysis and use of the clinical samples preved, the high expression of YAP and GLUT1 was closely related to the low survival of HCC patients. Multi-variate Cox proportional hazards analysis of clinical samples testified that the level of YAP and GLUT1 has important reference significance for predicting the stages of disease progression in HCC patients. We then put forward that YAP and GLUT1 have the potential to serve as novel biomarkers for the diagnosis and treatment of HCC and have an enormous potential in prognosis as well. The findings above might provide possible targets for the development of cancer targeted drugs and novel ideas for the therapy and aid in realization of the goal of both early detection and treatment of HCC.

We next explored how the inhibition of glucose uptake leads to changes in the cellular metabolic network, and its association with cell proliferation. Increased glucose uptake is the main characteristic of glucose metabolism in the tumor cells [53]. Glycolysis and oxidative phosphorylation are both important parts of the glucose metabolism. Seahorse energy metabolism experiments showed the lack of glucose caused by WZ35 restrained glycolysis and oxidative phosphorylation (Fig. 6a,g). The cancer cells are primarily dependent on glycolysis for energy production. Many intermediates of glycolysis can be transferred to various intermediate pathways to produce different biosynthetic precursors. Among them, 5-phosphate ribose, the structural component of nucleotides, is partially oxidized from 6-phosphate glucose in the first branch pathway, the pentose phosphate pathway (PPP) [54]. Additionally, a number of studies have found that the level of oxidative phosphorylation is closely related to the proliferation ability of the tumor 
cells. The intervention in the process of oxidative phosphorylation has a significant impact on the proliferation and metastasis of cancer both under in vivo and in vitro settings [55-56]. Purine metabolism is a type of nucleotide metabolism. Gaglio $D$ et.al found that generation of the nucleotide bases can rescue the proliferation of glutamine-deficient cells [57-58]. Metabolomics analysis showed the purine metabolism of HCC cells was restrained by WZ35. Combining the above evidence, we speculated that the inhibition of glucose causes glycolysis and oxidative phosphorylation reduction and then leads to an impaired purine metabolism and ultimatly decreases the proliferation activity of HCC cells (Fig. 6k). This finding demonstrates targeting cancer cell metabolism, especially glycometabolism, might be a potential method for HCC treatment.

\section{Conclusions}

In conclusion, our work first demonstrated that a potent small molecule YAP inhibitor WZ35 can restrain the proliferation of HCC through ROS-YAP-GLUT1 axis mediated glucose intake inhibition. YAP and GLUT1 has important reference significance for predicting the stages of disease progression in HCC patients and have the potential to serve as novel biomarkers for the diagnosis and treatment of HCC. Targeting the metabolic process of hepatoma carcinoma cells may be a hopeful way in tumor treatment. Our findings present novel insights into the pathology of HCC and potential targets for new therapeutic strategies. In the next step, we plan to further clarify the mechanism by which YAP regulates GLUT1, determine the specific binding site of TEAD and GLUT1 promoter, and explore the role of epigenetic changes such as histone methylation modification in activating the transcription of GLUT1.

\section{Abbreviations}

HCC: hepatocellular carcinoma;

YAP: yes-associated protein 1;

YAP1: Yes1 associated transcriptional regulator;

GLUT1: Glucose transporter 1;

TEM: Transmission electron microscope;

SLC2A1: solute carrier family 2;

ROS: reactive oxygen species;

OCR: oxygen consumption rate;

ECAR: extracellular acidification rate;

TCGA: The Cancer Genome Atlas; 
GEO: Gene Expression Omnibus;

ROC: receiver operating characteristic;

AUC: area under curve;

OS: overall survival;

ATP: adenosine triphosphate;

IHC: immunohistochemistry;

TNM: tumor node metastasis;

DAPI: 4',6-diamidino-2-phenylindole;

shRNA: short hairpin RNA;

GFP: green fluorescent protein;

FBS: fetal bovine serum;

PBS: phosphate-buffered saline;

SDS-PAGE: sodium dodecyl sulfate polyacrylamide gel electrophoresis;

SPF: specific-pathogen-free;

H\&E: hematoxylin and eosin;

DEG: different expression gene.

\section{Declarations}

\section{Ethics approval and consent to participate}

The ethical approval for this project including informed patient consent was granted following guidelines of the First Affiliated Hospital of Wenzhou Medical University Ethics Committee.

Procedures involving animals and their care was performed in accordance with the Declaration of Helsinki and approved by Wenzhou Medical University Ethics Committee.

\section{Consent for publication}

Informed consent was obtained from all individual participants included in the study.

\section{Availability of data and materials}


The datasets analyzed during the current study are not publicly available but are available from the corresponding author on reasonable request.

\section{Competing interests}

The authors declare no competing interests. The graphic was created with BioRender.com.

\section{Funding}

This work was supported by National Natural Science Foundation of China (No. 81902803), Project of Zhejiang Provincial Naturel Science Foundation of China (LY21H160057), the basic scientific research project of Wenzhou (Y20190055, Y20190085), the Medical and Health Science Project of Zhejiang Province (2020KY645) and Yuying project of The Second Affiliated Hospital and Yuying Children's Hospital of Wenzhou Medical University.

\section{Authors' contributions}

LHW, XKL, LHL and TKC conceived the idea and designed the research. ZZ, QL, YCT and JBC performed in vitro experiments. GWJ, YYZ, JCD and RXD performed mice xenograft and metabolism experiments. RXD, ZXW, LJZ,SYK analyzed patient samples. TKC, LHW and LHL analyzed the data. LHW, ZZ and QL wrote the manuscript. LHW, XKL and TKC revised the manuscript. The authors read and approved the final manuscript.

\section{Acknowledgements}

We would like to thank our colleagues from Laboratory Animal Centre of Wenzhou Medical University for their assistance.

\section{References}

1. Siegel RL, Miller KD, Jemal A. Cancer statistics, 2020. CA Cancer J Clin. 2020 Jan;70(1):7-30. doi: 10.3322/caac.21590. Epub 2020 Jan 8. PMID: 31912902.

2. Matthew, G, Vander, et al. Understanding the Warburg effect: the metabolic requirements of cell proliferation.[J]. Science (New York, N.Y.), 2009.

3. Vander Heiden MG, DeBerardinis RJ. Understanding the Intersections between Metabolism and Cancer Biology. Cell. 2017;168(4):657-669. doi:10.1016/j.cell.2016.12.039.

4. Chang $\mathrm{C} \mathrm{H}$, Jing Q, O'Sullivan D, et al. Metabolic Competition in the Tumor Microenvironment Is a Driver of Cancer Progression[J]. Cell, 2015, 162(6):1229-1241.

5. Poff A, Kourtnik A, Egan K M , et al. Targeting the Warburg effect for Cancer Treatment: Ketogenic Diets for Management of Glioma[J]. Seminars in Cancer Biology, 2017:S1044579X17301244.

6. Abdelwahab MG, Fenton KE, Preul MC, Rho JM, Lynch A, Stafford P, et al. The ketogenic diet is an effective adjuvant to radiation therapy for the treatment of malignant glioma. PLoS One. 
2012;7:e36197. doi: 10.137/journal.pone.0036197.

7. Goel A, Mathupala S P , Pedersen P L . Glucose Metabolism in Cancer[J]. Journal of Biological Chemistry, 2003, 278(17):15333-15340.

8. Seyfried TN, Sanderson TM, El-Abbadi MM, McGowan R, Mukherjee P. Role of glucose and ketone bodies in the metabolic control of experimental brain cancer. $\mathrm{Br} J$ Cancer. 2003;89:1375-82. doi: 10.038/sj.bjc.6601269.

9. Kobayashi Y, Banno K, H Kunitomi, et al. Warburg effect in Gynecologic cancers[J]. The Journal of Obstetrics and Gynaecology Research, 2019.

10. Deng D, Yan N. GLUT, SGLT, and SWEET: Structural and mechanistic investigations of the glucose transporters[J]. Protn ence, 2016, 25(3):546-558.

11. Kim W S , Kim Y Y, Jang S J , et al. Glucose transporter 1 (GLUT1) expression is associated with intestinal type of gastric carcinoma.[J]. Journal of Korean Medical ence, 2000, 15(4).

12. Shailendra, Kapoor. Glucose transporter 1 (GLUT1) and its emerging role as a significant prognostic marker in systemic malignancies[J]. International Journal of Colorectal Disease, 2013.

13. Xing, Tao, Kaishan, et al. RRAD inhibits aerobic glycolysis, invasion, and migration and is associated with poor prognosis in hepatocellular carcinoma[J]. Tumour biology, 2016, 37(4):5097-5105.

14. Kim W S『Kim Y Y『Jang S J『et al『Glucose transporter 1 ( GLUT1) expression is associated with

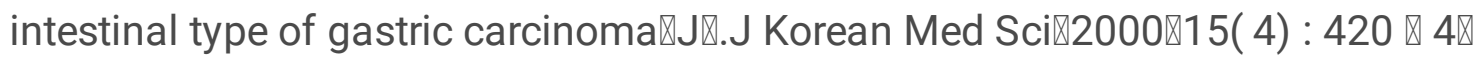

15. Mezheyeuski A , Alexander N, Tatjana B , et al. Inter- and intra-tumoral relationships between vasculature characteristics, GLUT1 and budding in colorectal carcinoma[J]. Histology and histopathology, 2015, 30(10):11613.

16. Facilitative glucose transporters: Implications for cancer detection, prognosis and treatment[J]. Metabolism-clinical \& Experimental, 2016, 65(2):124-139.

17. Wang. Inhibition of glucose transporter 1 (GLUT1) chemosensitized head and neck cancer cells to cisplatin.[J]. Technology in cancer research \& treatment, 2013.

18. Significance of Glucose Transporter Type 1 (GLUT-1) Expression in the Therapeutic Strategy for Pancreatic Ductal Adenocarcinoma[J]. Annals of Surgical Oncology, 2018.

19. Moya IM, Halder G. Hippo-YAP/TAZ signalling in organ regeneration and regenerative medicine. Nat Rev Mol Cell Biol. 2019 Apr;20(4):211-226. doi: 10.1038/s41580-018-0086-y. PMID: 30546055.

20. Piccolo S, Dupont S, Cordenonsi M. The biology of YAP/TAZ: hippo signaling and beyond. Physiol Rev. 2014 Oct;94(4):1287-312. doi: 10.1152/physrev.00005.2014. PMID: 25287865.

21. Dong J, Feldmann G, Huang J, Wu S, Zhang N, Comerford SA, Gayyed MF, Anders RA, Maitra A, Pan D. Elucidation of a universal size-control mechanism in Drosophila and mammals. Cell. $2007 \mathrm{Sep}$ 21;130(6):1120-33. doi: 10.1016/j.cell.2007.07.019. PMID: 17889654; PMCID: PMC2666353.

22. Cao X, Pfaff SL, Gage FH. YAP regulates neural progenitor cell number via the TEA domain transcription factor. Genes Dev. 2008 Dec 1;22(23):3320-34. doi: 10.1101/gad.1726608. Epub 2008 Nov 17. PMID: 19015275; PMCID: PMC2600760. 
23. Hanahan D, Weinberg RA. Hallmarks of cancer: the next generation. Cell. 2011 Mar 4;144(5):646-74. doi: 10.1016/j.cell.2011.02.013. PMID: 21376230.

24. Wang $L$, Han $L$, Tao $Z$, et al. The curcumin derivative WZ35 activates ROS-dependent JNK to suppress hepatocellular carcinoma metastasis[J]. Food \& Function, 2018, 9.

25. Chen $T$, Zhao $L$, Chen $S$, et al. The curcumin analogue WZ35 affects glycolysis inhibition of gastric cancer cells through ROS-YAP-JNK pathway[J]. Food and Chemical Toxicology, 2020, 137:111131-.

26. Wang $L$, Zhu Z , Han $L$, et al. A curcumin derivative, WZ35, suppresses hepatocellular cancer cell growth via downregulating YAP-mediated autophagy[J]. Food \& Function, 2019, 10.

27. Wang $L$, Wang $C$, Tao $Z$, et al. Curcumin derivative WZ35 inhibits tumor cell growth via ROS-YAPJNK signaling pathway in breast cancer[J]. Journal of Experimental \& Clinical Cancer Research, 2019, 38(1).

28. Yap regulates glucose utilization and sustains nucleotide synthesis to enable organ growth[J]. Embo Journal, 2017.

29. Zou P, Zhang JR, Xia YQ, Kanchana K, Guo GL, Chen WB, Huang Y, Wang Z, Yang SL, Liang G. ROS generation mediates the anti-cancer effects of WZ35 via activating JNK and ER stress apoptotic pathways in gastric cancer. Oncotarget. 2015;6:5860-5876.

30. Fornes O, Castro-Mondragon JA, Khan A, van der Lee R, Zhang X, Richmond PA, Modi BP, Correard S, Gheorghe M, Baranašić D, Santana-Garcia W, Tan G, Chèneby J, Ballester B, Parcy F, Sandelin A, Lenhard B, Wasserman WW, Mathelier A. JASPAR 2020: update of the open-access database of transcription factor binding profiles. Nucleic Acids Res. 2020 Jan 8;48(D1):D87-D92. doi: 10.1093/nar/gkz1001. PMID: 31701148; PMCID: PMC7145627.

31. Haeussler M, Zweig AS, Tyner C, Speir ML, Rosenbloom KR, Raney BJ, Lee CM, Lee BT, Hinrichs AS, Gonzalez JN, Gibson D, Diekhans M, Clawson H, Casper J, Barber GP, Haussler D, Kuhn RM, Kent WJ. The UCSC Genome Browser database: 2019 update. Nucleic Acids Res. 2019 Jan 8;47(D1):D853D858. doi: 10.1093/nar/gky1095. PMID: 30407534; PMCID: PMC6323953.

32. Gutta C, Rahman A, Aura C, Dynoodt P, Charles EM, Hirschenhahn E, Joseph J, Wouters J, de Chaumont C, Rafferty M, Warren M, van den Oord JJ, Gallagher WM, et al. Low expression of proapoptotic proteins Bax, Bak and Smac indicates prolonged progression-free survival in chemotherapy-treated metastatic melanoma. Cell Death Dis. 2020; 11: 124.

33. Tong H, Zhang X, Meng X, Lu L, Mai D, Qu S. Simvastatin Inhibits Activation of NADPH Oxidase/p38 MAPK Pathway and Enhances Expression of Antioxidant Protein in Parkinson Disease Models. Front Mol Neurosci. 2018; 11: 165.

34. Moroishi T, Hansen CG, Guan KL. The emerging roles of YAP and TAZ in cancer. Nat Rev Cancer. 2015 Feb;15(2):73-79. doi: 10.1038/nrc3876. Epub 2015 Jan 16. PMID: 25592648; PMCID: PMC4562315.

35. Zanconato F, Cordenonsi M, Piccolo S. YAP/TAZ at the Roots of Cancer. Cancer Cell. 2016 Jun 13;29(6):783-803. doi: 10.1016/j.ccell.2016.05.005. PMID: 27300434; PMCID: PMC6186419. 
36. Tunable Hybrid Matrices Drive Epithelial Morphogenesis and YAP Translocation[J]. Advanced Science, 2020.

37. Moroishi T, Hansen CG, Guan KL. The emerging roles of YAP and TAZ in cancer. Nat Rev Cancer. 2015 Feb;15(2):73-79. doi: 10.1038/nrc3876. Epub 2015 Jan 16. PMID: 25592648; PMCID: PMC4562315.] [Zanconato F, Cordenonsi M, Piccolo S. YAP/TAZ at the Roots of Cancer. Cancer Cell. 2016 Jun 13;29(6):783-803. doi: 10.1016/j.ccell.2016.05.005. PMID: 27300434; PMCID: PMC6186419.

38. Filomeni G, De Zio D, Cecconi F. Oxidative stress and autophagy: the clash between damage and metabolic needs. Cell Death Differ. 2015 Mar;22(3):377-88. doi: 10.1038/cdd.2014.150. Epub 2014 Sep 26. PMID: 25257172; PMCID: PMC4326572.

39. Cairns RA, Harris IS, Mak TW: Regulation of cancer cell metabolism. Nat Rev Cancer 2011; 11: 85-95.

40. Sun Linchong,Suo Caixia,Li Shi-Ting et al. Metabolic reprogramming for cancer cells and their microenvironment: Beyond the Warburg Effect.[J] .Biochim Biophys Acta Rev Cancer, 2018.

41. Singal AG, Pillai A, Tiro J. Early detection, curative treatment, and survival rates for hepatocellular carcinoma surveillance in patients with cirrhosis: a meta-analysis. PLoS Med. 2014 Apr 1;11(4):e1001624. doi: 10.1371/journal.pmed.1001624. PMID: 24691105; PMCID: PMC3972088.

42. Singal AG, Pillai A, Tiro J. Early detection, curative treatment, and survival rates for hepatocellular carcinoma surveillance in patients with cirrhosis: a meta-analysis. PLoS Med. 2014 Apr 1;11(4):e1001624. doi: 10.1371/journal.pmed.1001624. PMID: 24691105; PMCID: PMC3972088.

43. Pavlova NN, Thompson CB. The Emerging Hallmarks of Cancer Metabolism. Cell Metab. 2016 Jan 12;23(1):27-47. doi: 10.1016/j.cmet.2015.12.006. PMID: 26771115; PMCID: PMC4715268.

44. Koppenol WH, Bounds PL, Dang CV. Otto Warburg's contributions to current concepts of cancer metabolism. Nat Rev Cancer. 2011 May;11(5):325-37. doi: 10.1038/nrc3038. Epub 2011 Apr 14. Erratum in: Nat Rev Cancer. 2011 Aug;11(8):618. PMID: 21508971.

45. Pietrocola F, Pol J, Vacchelli E, Rao S, Enot DP, Baracco EE, Levesque S, Castoldi F, Jacquelot N, Yamazaki T, Senovilla L, Marino G, Aranda F, Durand S, Sica V, Chery A, Lachkar S, Sigl V, Bloy N, Buque A, Falzoni S, Ryffel B, Apetoh L, Di Virgilio F, Madeo F, Maiuri MC, Zitvogel L, Levine B, Penninger JM, Kroemer G. Caloric Restriction Mimetics Enhance Anticancer Immunosurveillance. Cancer Cell. 2016 Jul 11;30(1):147-160. doi: 10.1016/j.ccell.2016.05.016. PMID: 27411589; PMCID: PMC5715805.

46. Nencioni A, Caffa I, Cortellino S, Longo VD. Fasting and cancer: molecular mechanisms and clinical application. Nat Rev Cancer. 2018;18(11):707-719. doi:10.1038/s41568-018-0061-0

47. Faiena I, Ueno D, Shuch B. Glutamine and the Tumor Immune Microenvironment. Eur Urol. 2019 May;75(5):764-765. doi: 10.1016/j.eururo.2019.01.015. Epub 2019 Jan 30. PMID: 30711333.

48. Green R. Vitamin B12 deficiency from the perspective of a practicing hematologist. Blood. 2017 May 11;129(19):2603-2611. doi: 10.1182/blood-2016-10-569186. Epub 2017 Mar 30. PMID: 28360040.Moya IM, Halder G. Hippo-YAP/TAZ signalling in organ regeneration and regenerative 
medicine. Nat Rev Mol Cell Biol. 2019 Apr;20(4):211-226. doi: 10.1038/s41580-018-0086-y. PMID: 30546055.

49. Moya IM, Halder G. Hippo-YAP/TAZ signalling in organ regeneration and regenerative medicine. Nat Rev Mol Cell Biol. 2019 Apr;20(4):211-226. doi: 10.1038/s41580-018-0086-y. PMID: 30546055.

50. Wang L, Luo JY, Li B, Tian XY, Chen LJ, Huang Y, Liu J, Deng D, Lau CW, Wan S, Ai D, Mak KK, Tong KK, Kwan KM, Wang N, Chiu JJ, Zhu Y, Huang Y. Integrin-YAP/TAZ-JNK cascade mediates atheroprotective effect of unidirectional shear flow. Nature. 2016 Dec 22;540(7634):579-582. doi: 10.1038/nature20602. Epub 2016 Dec 7. PMID: 27926730.

51. Dey A, Varelas X, Guan KL. Targeting the Hippo pathway in cancer, fibrosis, wound healing and regenerative medicine. Nat Rev Drug Discov. 2020;19(7):480-494. doi:10.1038/s41573-020-0070-z.

52. Barski A, Cuddapah S, Cui K, Roh TY, Schones DE, Wang Z, Wei G, Chepelev I, Zhao K. High-resolution profiling of histone methylations in the human genome. Cell. 2007 May 18;129(4):823-37. doi: 10.1016/j.cell.2007.05.009. PMID: 17512414.

53. Ganapathy-Kanniappan S, Geschwind JF. Tumor glycolysis as a target for cancer therapy: progress and prospects. Mol Cancer. 2013;12:152. Published 2013 Dec 3. doi:10.1186/1476-4598-12-152

54. Wang R, Dillon CP, Shi LZ, Milasta S, Carter R, Finkelstein D, McCormick LL, Fitzgerald P, Chi H, Munger J, Green DR. The transcription factor Myc controls metabolic reprogramming upon T lymphocyte activation. Immunity. 2011 Dec 23;35(6):871-82. doi: 10.1016/j.immuni.2011.09.021. PMID: 22195744; PMCID: PMC3248798.

55. Thakur S, Daley B, Gaskins K, Vasko VV, Boufraqech M, Patel D, Sourbier C, Reece J, Cheng SY, Kebebew E, Agarwal S, Klubo-Gwiezdzinska J. Metformin Targets Mitochondrial Glycerophosphate Dehydrogenase to Control Rate of Oxidative Phosphorylation and Growth of Thyroid Cancer In Vitro and In Vivo. Clin Cancer Res. 2018 Aug 15;24(16):4030-4043. doi: 10.1158/1078-0432.CCR-17-3167. Epub 2018 Apr 24. PMID: 29691295; PMCID: PMC6095745.

56. LeBleu VS, O'Connell JT, Gonzalez Herrera KN, Wikman H, Pantel K, Haigis MC, de Carvalho FM, Damascena A, Domingos Chinen LT, Rocha RM, Asara JM, Kalluri R. PGC-1 a mediates mitochondrial biogenesis and oxidative phosphorylation in cancer cells to promote metastasis. Nat Cell Biol. 2014 Oct;16(10):992-1003, 1-15. doi: 10.1038/ncb3039. Epub 2014 Sep 21. Erratum in: Nat Cell Biol. 2014 Nov;16(11):1125. PMID: 25241037; PMCID: PMC4369153.

57. Gaglio D, Soldati C, Vanoni M, Alberghina L, Chiaradonna F. Glutamine deprivation induces abortive s-phase rescued by deoxyribonucleotides in k-ras transformed fibroblasts. PLoS One. 2009;4(3):e4715. doi: 10.1371/journal.pone.0004715. Epub 2009 Mar 5. PMID: 19262748; PMCID: PMC2650790.

58. Lunt SY, Muralidhar V, Hosios AM, Israelsen WJ, Gui DY, Newhouse L, Ogrodzinski M, Hecht V, Xu K, Acevedo PN, Hollern DP, Bellinger G, Dayton TL, Christen S, Elia I, Dinh AT, Stephanopoulos G, Manalis SR, Yaffe MB, Andrechek ER, Fendt SM, Vander Heiden MG. Pyruvate kinase isoform expression alters nucleotide synthesis to impact cell proliferation. Mol Cell. 2015 Jan 8;57(1):95-107. doi: 10.1016/j.molcel.2014.10.027. Epub 2014 Dec 4. PMID: 25482511; PMCID: PMC4289430. 


\section{Tables}

Table 1 Univariate and multivariate analyses of factors associated with HCC patient overall survival

\begin{tabular}{|c|c|c|c|c|c|c|c|c|}
\hline \multirow[t]{2}{*}{ Variables } & \multicolumn{4}{|c|}{ Univariate analysis } & \multicolumn{4}{|c|}{ Multivariate analysis } \\
\hline & RRb & SE & $95 \%$ & $P$ value & RRb & SE & $95 \%$ & $P$ value \\
\hline YAP & 1.819 & 0.233 & $\begin{array}{l}1.151- \\
2.875\end{array}$ & 0.01 & 1.717 & 0.235 & $\begin{array}{l}1.083- \\
2.721\end{array}$ & 0.021 \\
\hline Age & 1.014 & 0.01 & $\begin{array}{l}0.995- \\
1.033\end{array}$ & 0.159 & - & - & - & 0.081 \\
\hline Gender & 1.215 & 0.255 & $\begin{array}{l}0.737- \\
2.002\end{array}$ & 0.444 & - & - & - & 0.324 \\
\hline Tumor nodule & 0.259 & 1.113 & $\begin{array}{l}0.032- \\
2.077\end{array}$ & 0.045 & 0.49 & 0.322 & $\begin{array}{l}0.261- \\
0.922\end{array}$ & 0.027 \\
\hline Lymph node metastases & 0.899 & 0.232 & $\begin{array}{l}0.570- \\
1.416\end{array}$ & 0.645 & - & - & - & 0.762 \\
\hline HBV & 0.985 & 0.149 & $\begin{array}{l}0.736- \\
1.318\end{array}$ & 0.917 & - & - & - & 0.724 \\
\hline Venous invasion & 0.618 & 0.152 & $\begin{array}{l}0.459- \\
0.832\end{array}$ & 0.002 & 0.414 & 0.305 & $\begin{array}{l}0.227- \\
0.752\end{array}$ & 0.004 \\
\hline Liver cirrhosis & 0.812 & 0.178 & $\begin{array}{l}0.573- \\
1.150\end{array}$ & 0.241 & - & - & - & 0.550 \\
\hline
\end{tabular}

YAP: yes-associated protein 1;

HCC: hepatocellular carcinoma;

HBV: hepatitis B virus;

SE: standard error

Figures 
A
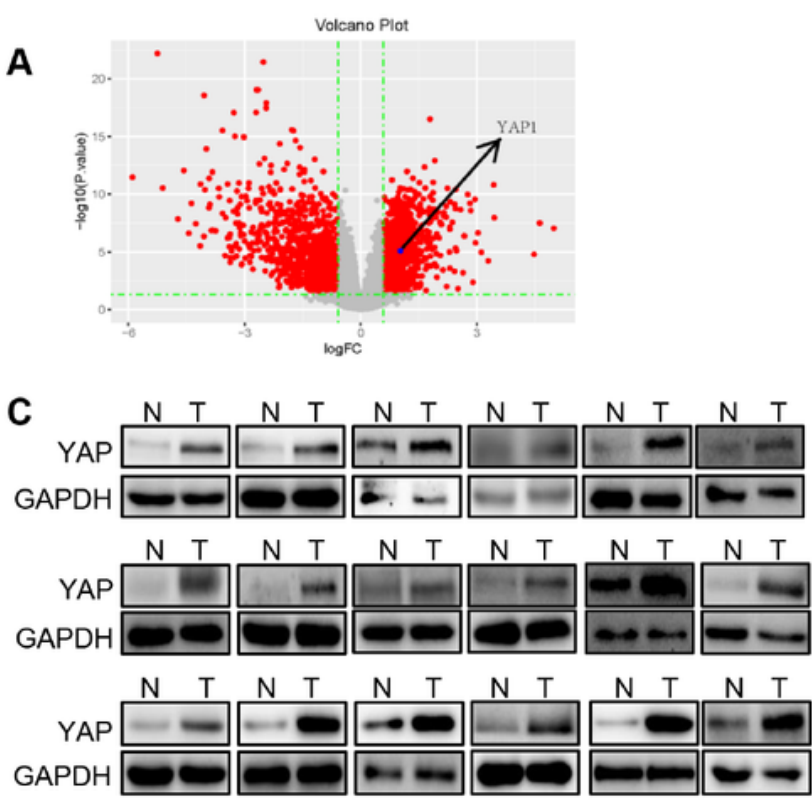

D

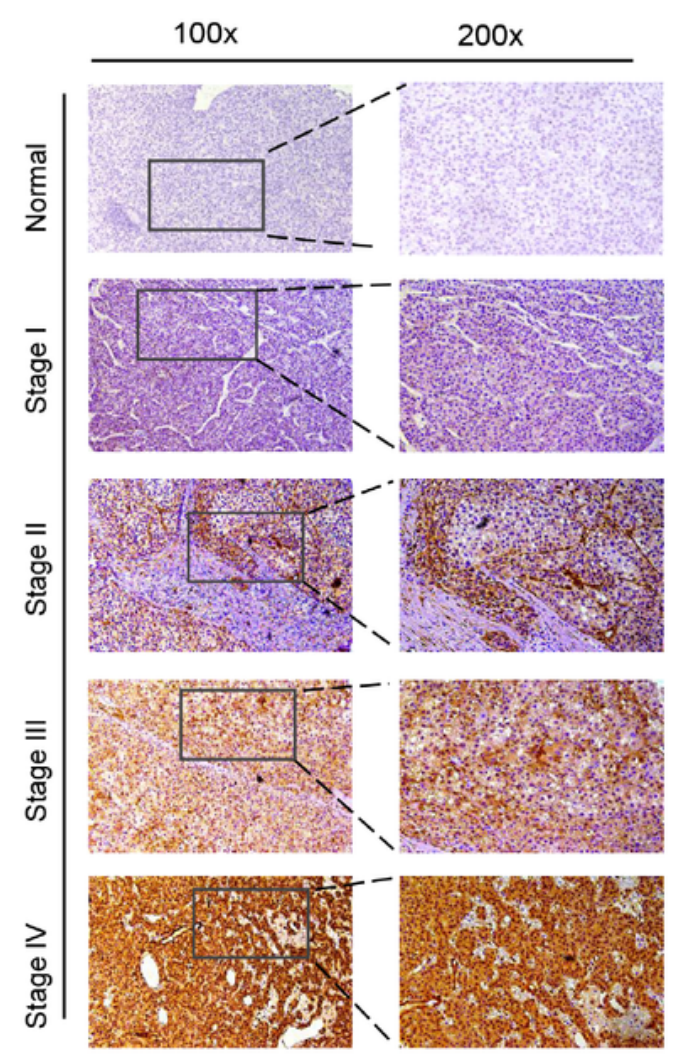

B
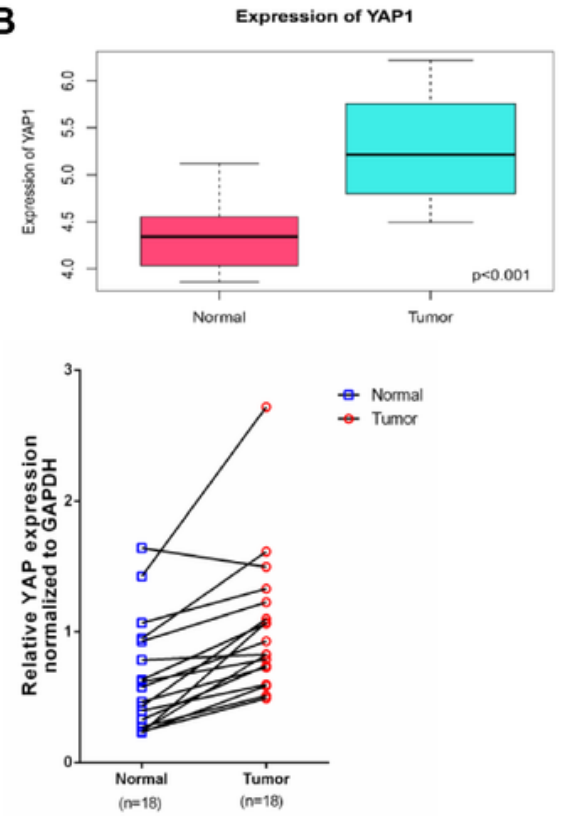

E

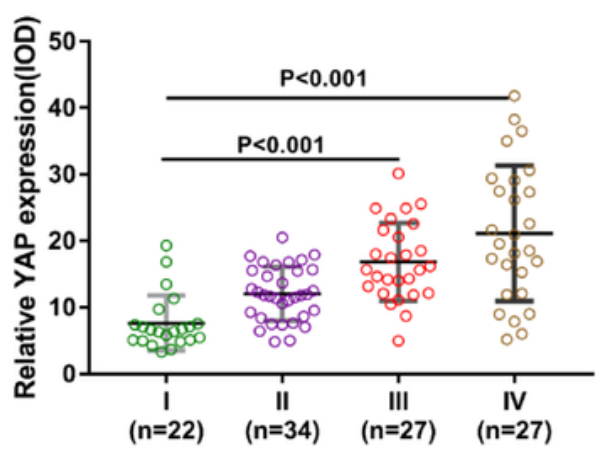

$\mathbf{F}$

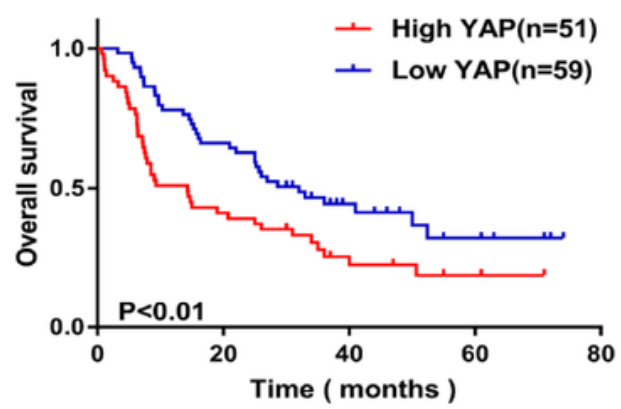

\section{Figure 1}

YAP upregulation in HCC corresponds to the poorer patient outcomes. a Volcano map of RNA-seq data from GEO database visualizing differentially expressed genes (DEGs) between the tumor samples and the normal ones with gene YAP1 marked out (Fold change of $>2$ and $P<<0.001$ ). b Box plot exhibits the distinct expression of YAP1 in the normal and the tumor samples, with the blue box is representative of the tumor samples and the red box of the normal samples. c Western blotting shows differential 
expression of YAP in the normal and the tumor tissues. d,e YAP IHC staining in HCC patient samples. Representative YAP-stained images of normal, TNM stage I, II, III and IV HCC tumor tissues have been shown and quantified. $f$ Kaplan-Meier approach visualized the relationship between YAP expression and OS in HCC patients $(n=110, P<0.01)$.

A

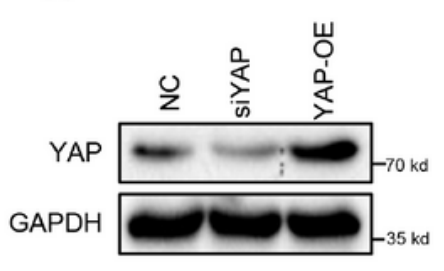

C

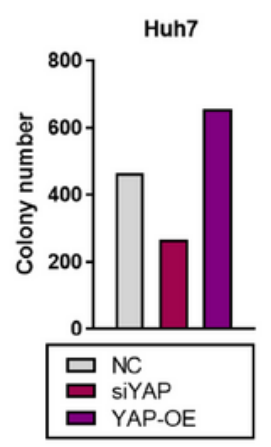

E

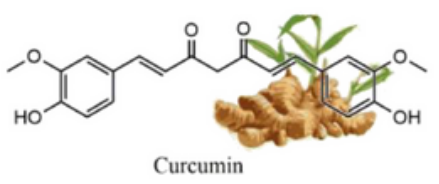

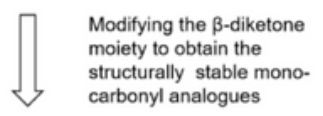<smiles>COc1cc(/C=C/C(=O)/C=C/c2ccccc2[N+](=O)[O-])ccc1O</smiles>

G

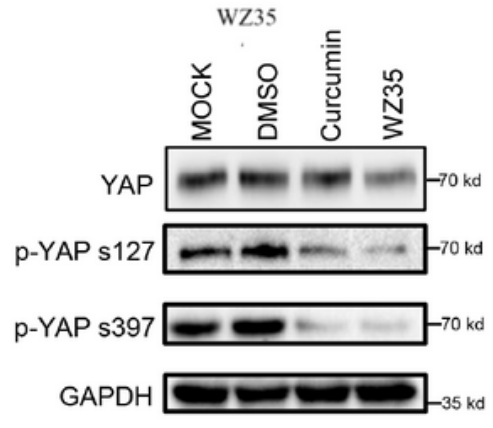

B
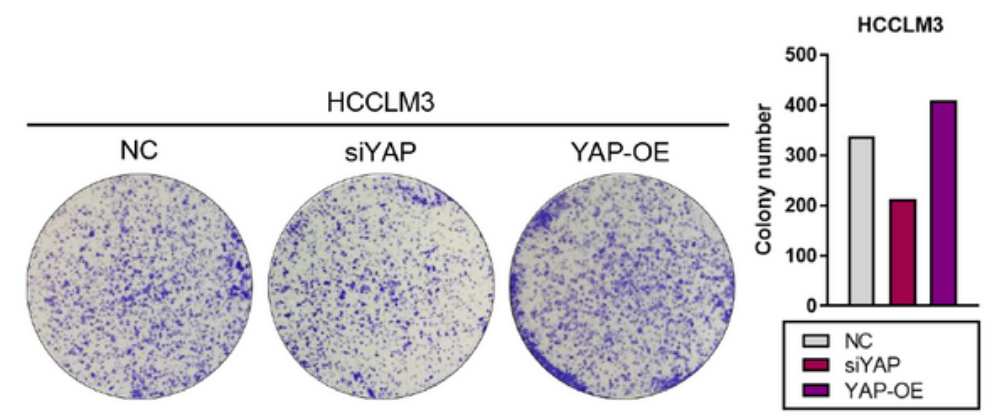

D
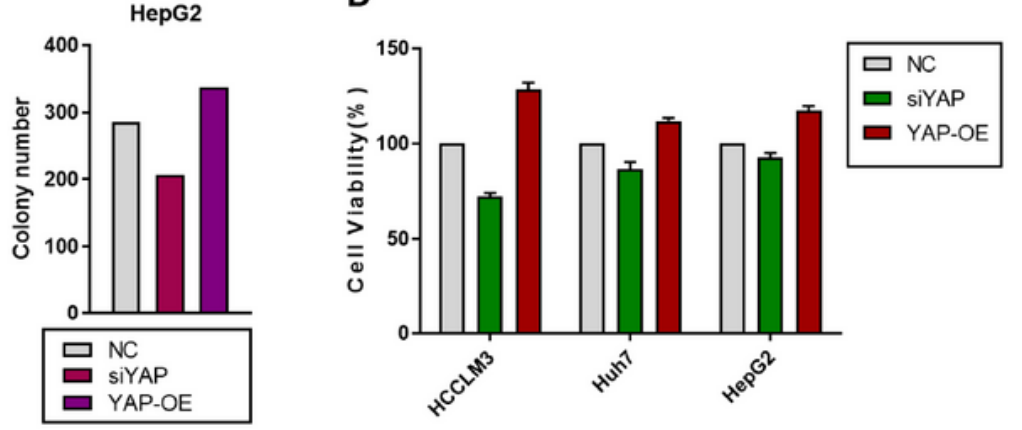

F Merged

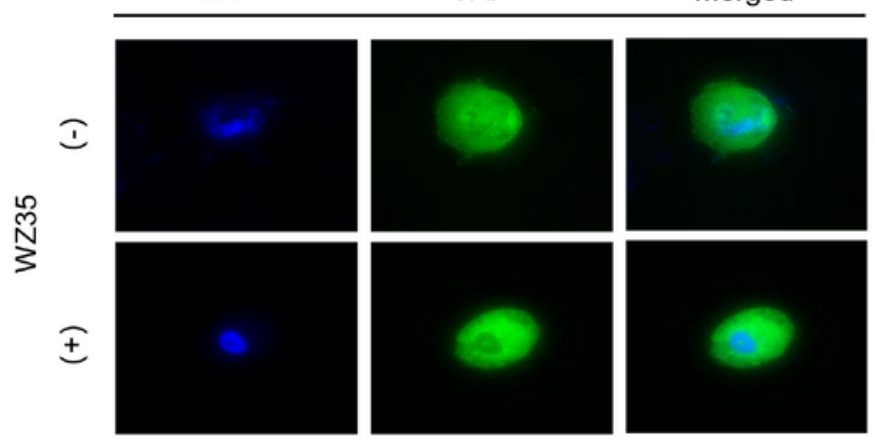

Figure 2 
WZ35 can inhibit YAP from entering the nucleus a HCCLM3 cell line was transfected with shRNA or pcDNA/peGFP vectors and harvested $48 \mathrm{~h}$ post-transfection to quantify the protein level of YAP and GAPDH. b,c Colony formation assay of different cell lines showed significant changes in stably transfected cells with representative images being shown and colony numbers being quantified. $d$ CCK-8 assays was performed after HCC cell lines were transfected with YAP shRNA or pcDNA/peGFP vectors. e The structure and synthesis of WZ35. $f$ Representative immunofluorescent images were displayed with bars of $10 \mu \mathrm{m}$, confirming the reduction of nuclear localization of YAP protein. (Green: YAP; Blue: DAPI). $g$ Western blotting analysis of the protein level of YAP, p-YAP s127 and p-YAP s397 in HCCLM3 cells treated with DMSO, curcumin and WZ35. 
A

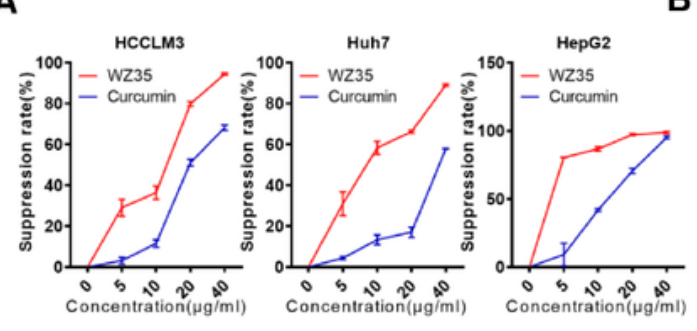

C

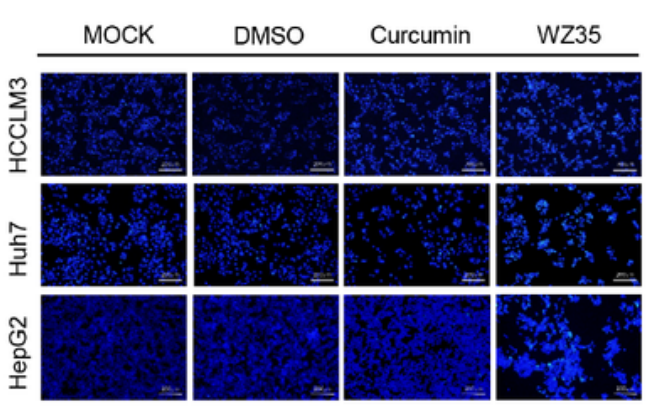

E
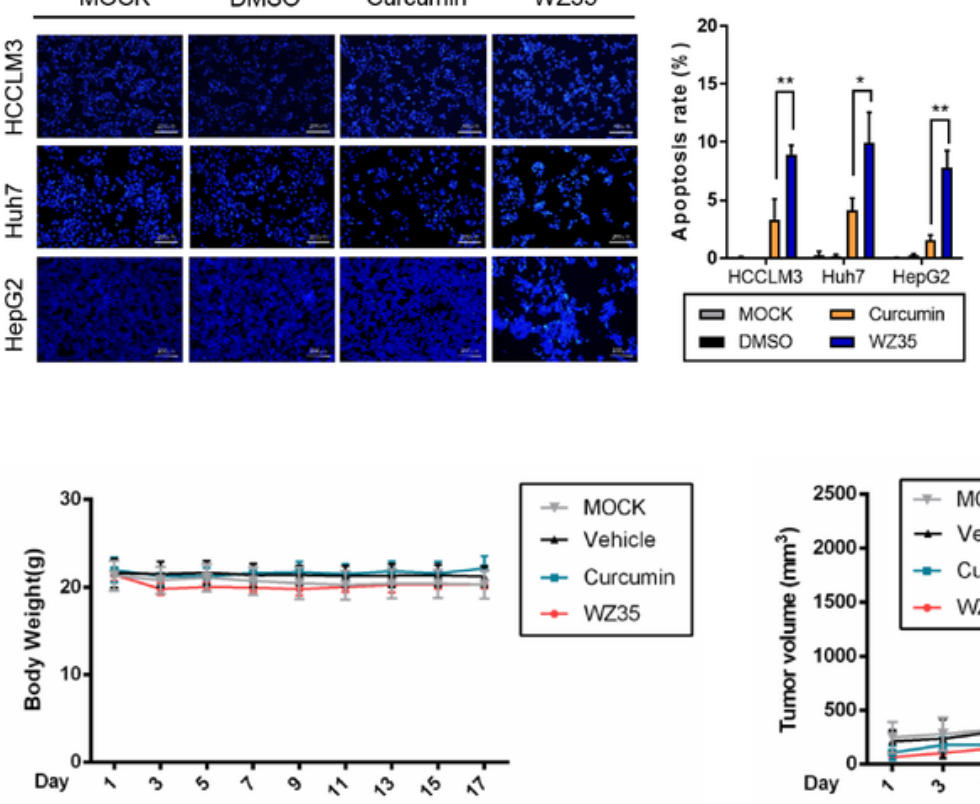

B
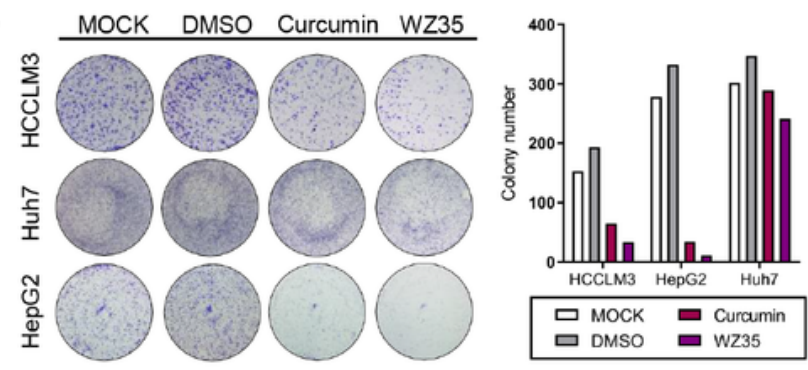

D

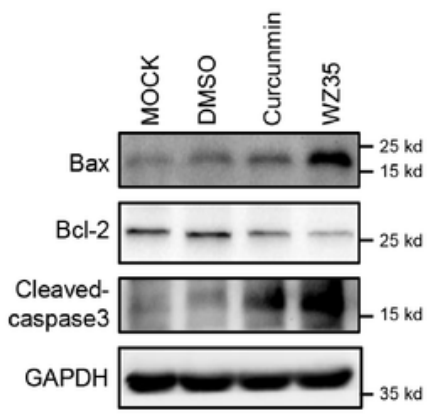

$\mathbf{F}$
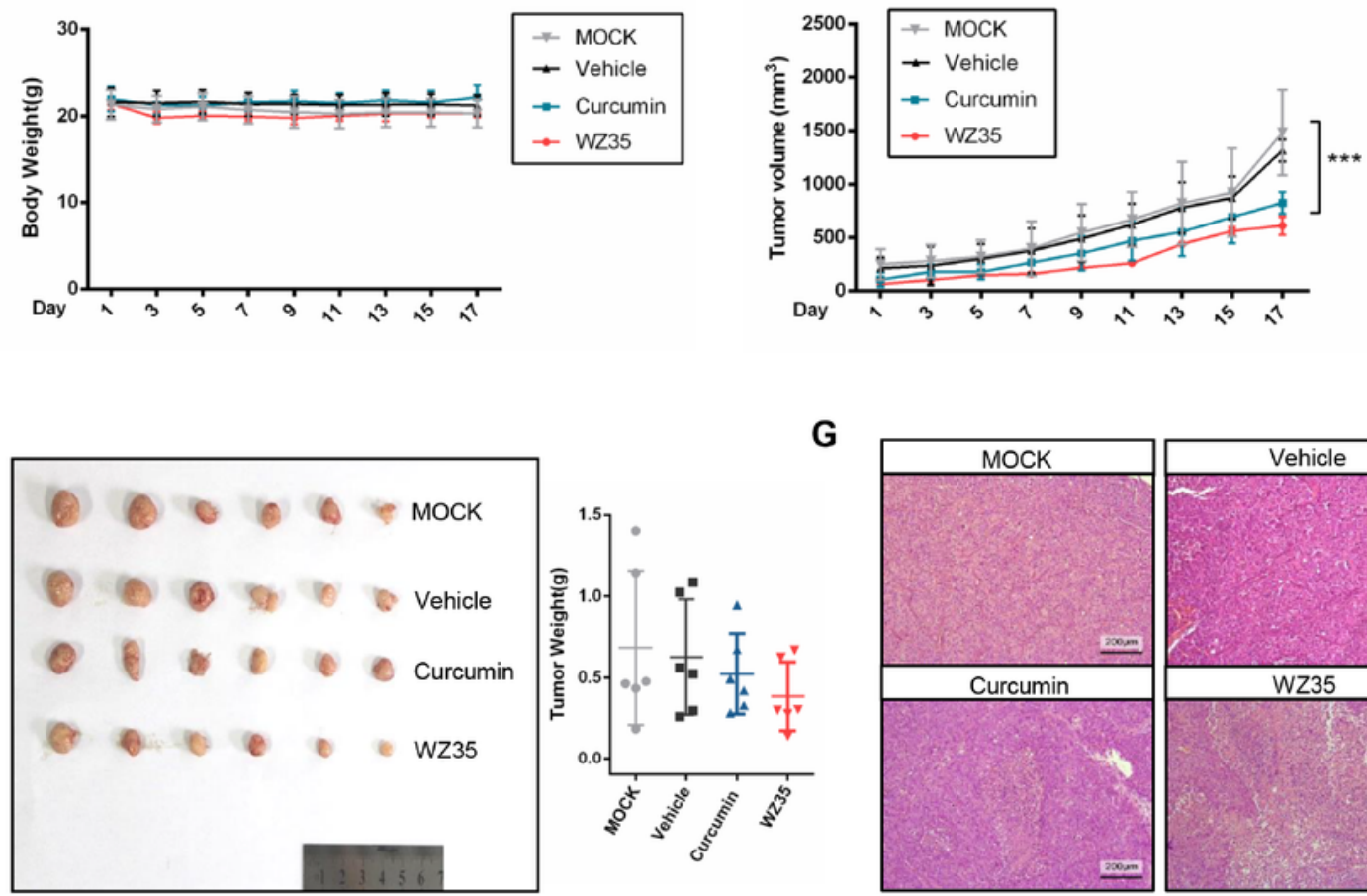

G
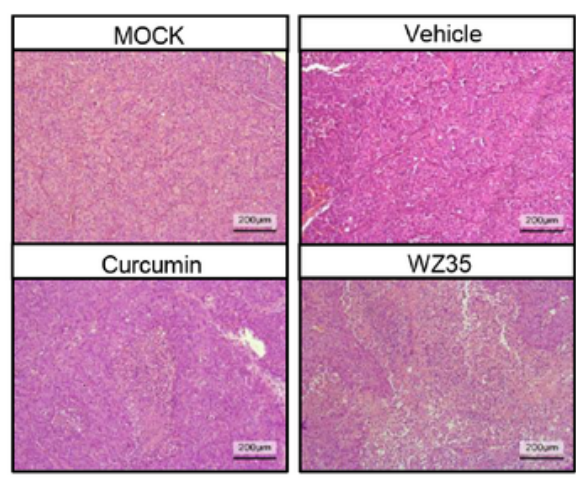

\section{Figure 3}

WZ35 exhibits significant antitumor activity a CCK-8 assays were utilized to measure the suppression rate of curcumin and WZ35 (with the concentration of $0,5,10,20$ to $40 \mu \mathrm{g} / \mathrm{mL}$ ) treated HCCLM3, HepG2, and Huh7 cells. b Colony formation assays of HCCLM3, HepG2, and Huh7 cells were conducted by plating 5000 indicated cells per well in 6-well plates to measure the effect of curcumin and WZ35 on clonogenicity with representative images and quantification of colony numbers being shown. c 
Representative fluorescence microscopy displaying nuclear transformations in HCCLM3, HepG2, and Huh7 cells following $18 \mathrm{~h}$ treatment of WZ35 $(10 \mu \mathrm{g} / \mathrm{mL})$ or treatment of curcumin $(10 \mu \mathrm{g} / \mathrm{mL})$ by means of DAPI staining. Results are presented as the mean \pm standard error from independent experiments performed in triplicate. ${ }^{*} P<0.05,{ }^{*} P<0.01$, student's $t$ test. $d$ The levels of various apoptosis associated proteins, including Bax, Bcl-2, and Cleaved caspase- 3 were detected by western blotting following the $18 \mathrm{~h}$ treatment of WZ35, with GAPDH serving as an internal control. e Quantitation of the murine body weight and tumor volumes over time in four indicated tumor xenograft groups, as measured by utilizing electronic balance and caliper. The results are presented as the mean \pm standard error from independent experiments in triplicate. ${ }^{*} \mathrm{P}<0.05,{ }^{\star \star} \mathrm{P}<0.01,{ }^{\star \star *} \mathrm{P}<0.001$, student's $\mathrm{t}$ test. $f$ Subcutaneous tumor xenograft models of WZ35-treated HCCLM3 were used. Mice were put to death by cervical dislocation and the tumors were harvested after 17 days. Optical image of each tumor and the weight curve was photographed and quantified, respectively. ( $n=6$ mice per group). $g$ The representative images of Hematoxylin and eosin staining of indicated treated tumors have been shown. Scale bars, $200 \mu \mathrm{m}$ for images. 
A

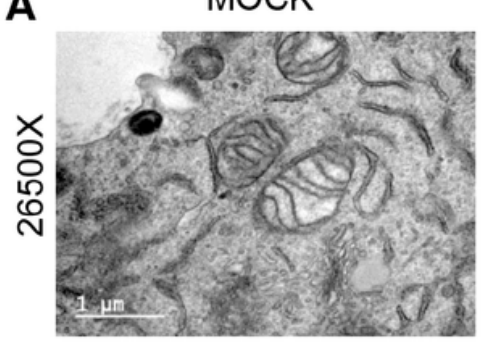

DMSO

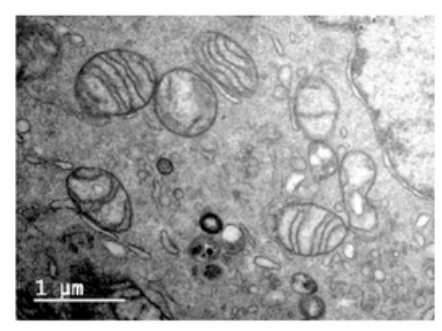

Curcumin

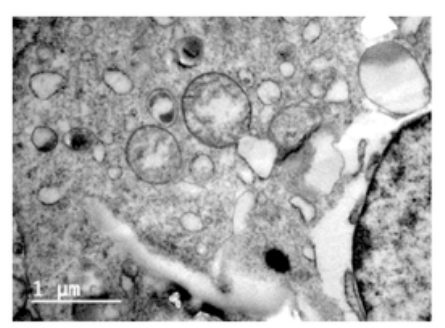

WZ35

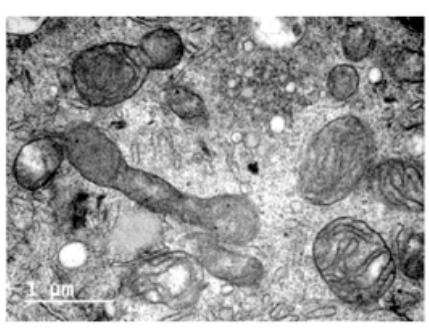

B

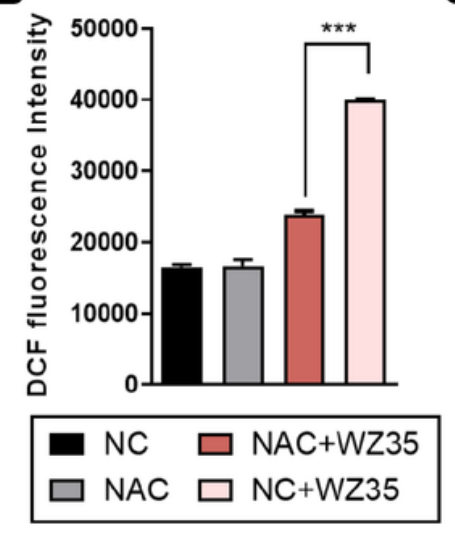

E

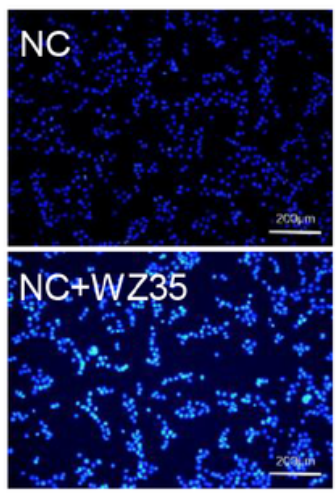

C

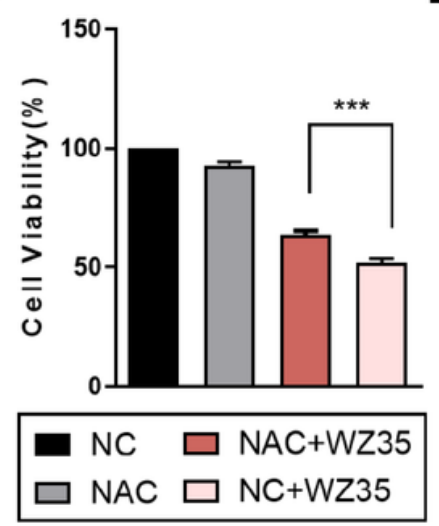

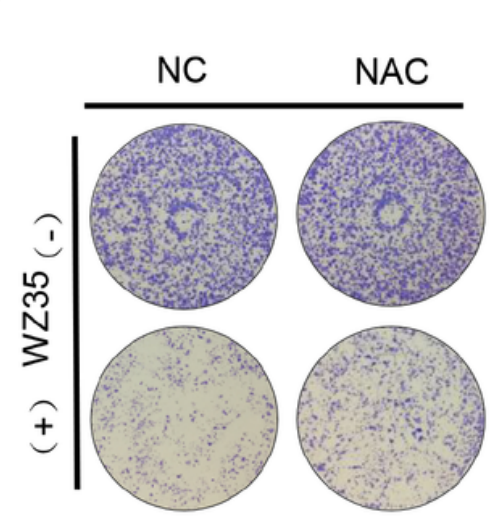

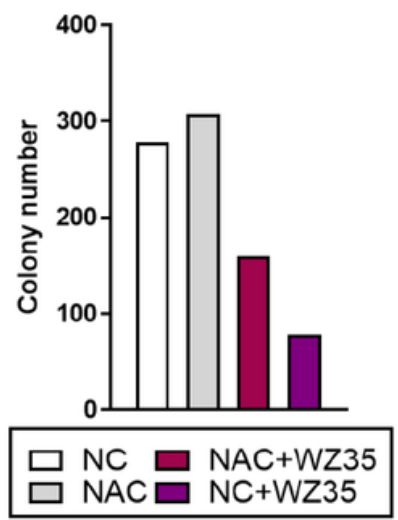

$\mathbf{F}$
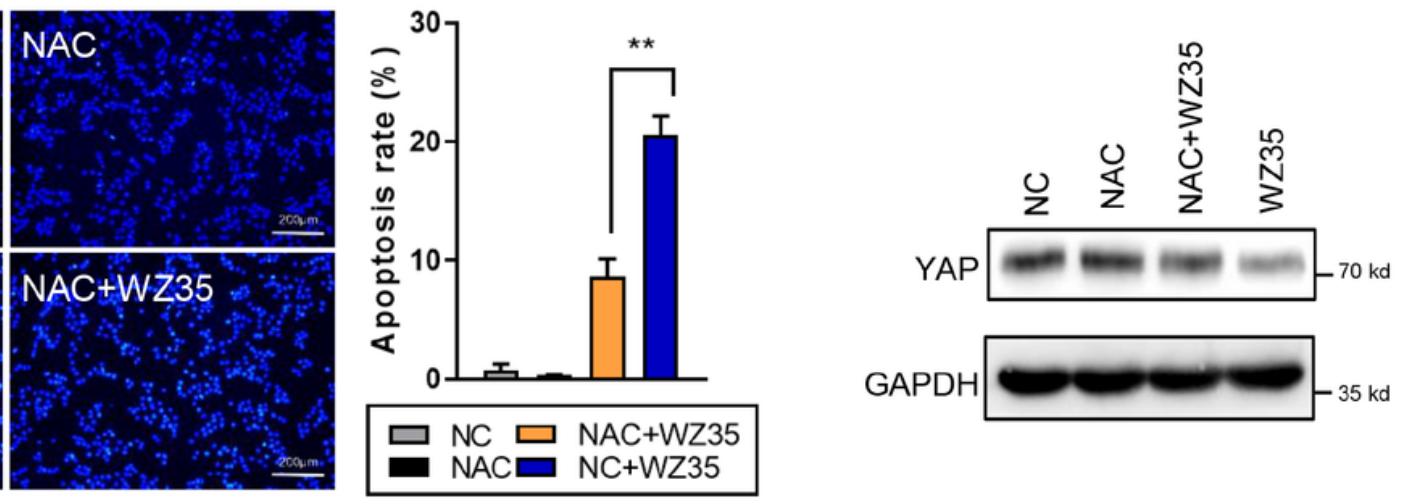

Figure 4

WZ35 inhibits growth of HCC cells in a ROS-dependent manner a Transmission electron micrographic (TEM) imaging showed changes in the morphology of HCCLM3 cells subsequent to the treatment of curcumin $(10 \mu \mathrm{g} / \mathrm{mL})$ or WZ35 $(10 \mu \mathrm{g} / \mathrm{mL})$ respectively. b The levels of intracellular reactive oxygen species (ROS) were determined by measuring the mean fluorescence intensity (MFI) of DCFH-DA (DCF) via flow cytometry in control or WZ35-treated HCCLM3 cells following pretreatment with NAC. Results are presented as the mean \pm standard error from independent experiments in triplicate. ${ }^{* \star} \mathrm{P}<0.001$, student's $\mathrm{t}$ test. c,d,e HCCLM3 cells with or without NAC $(5 \mathrm{mM})$ pretreatment were incubated with or without WZ35 $(10 \mu \mathrm{g} / \mathrm{mL})$ to assess the cellular proliferation and apoptosis via CCK-8 assays (c), colony formation 
assays (d) and DAPI staining assays (e) with representative images and/or quantifications have been shown. All these results are presented as the mean \pm standard error from independent experiments in triplicate. ${ }^{* \star P} \mathrm{P}<0.01,{ }^{\star \star *} \mathrm{P}<0.001$, student's $\mathrm{t}$ test. $\mathrm{f}$ Western blotting analysis of the YAP protein level of HCCLM3 cells treated with WZ35 or NAC.
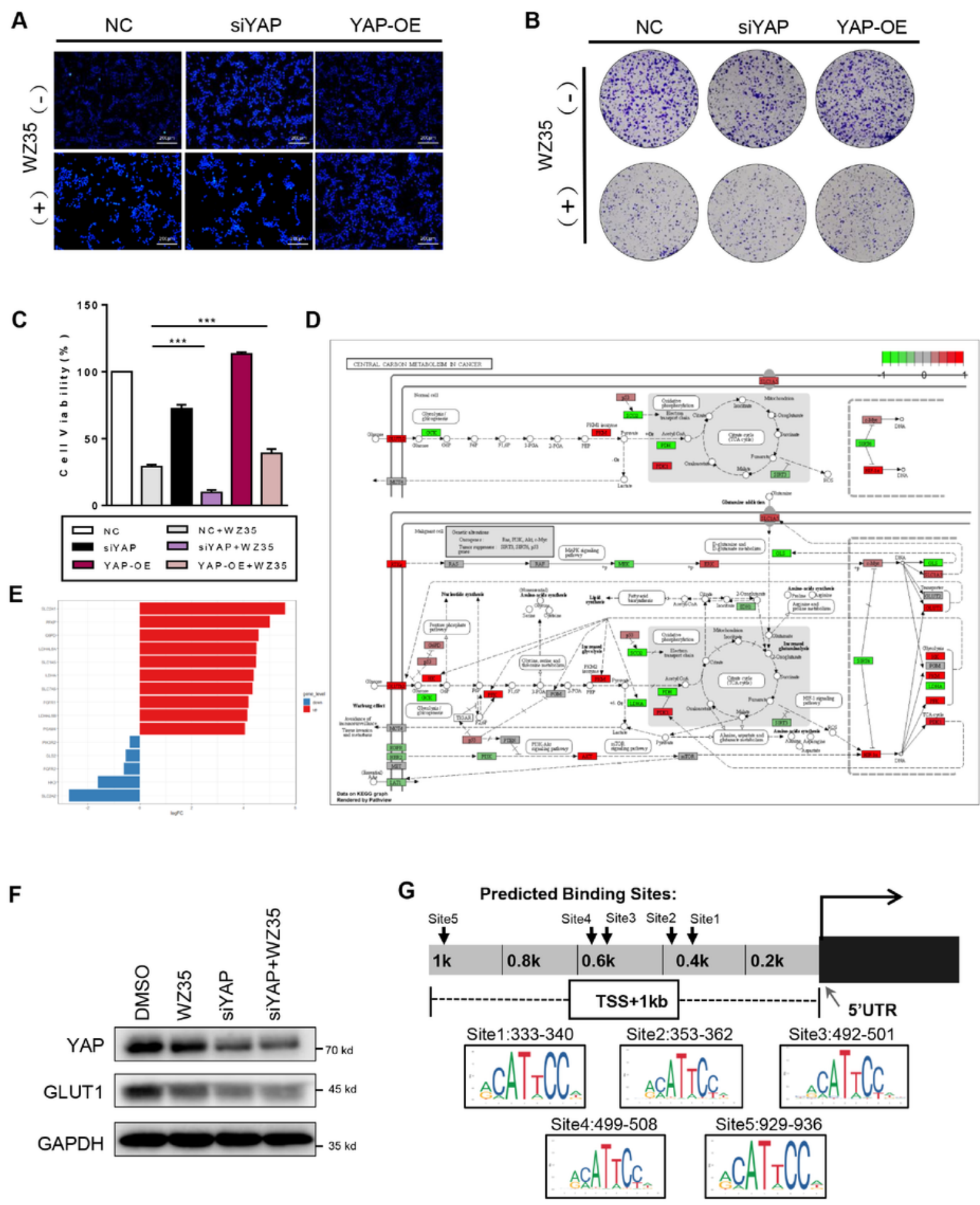

Figure 5 
WZ35 antitumor activity depends upon inhibition of the YAP protein and allows it to regulate the expression of GLUT1 a,b,c DAPI staining assays a colony formation assays (b) and CCK-8 assays (c) were conducted to measure apoptosis and cellular viability in control or WZ35-treated $(10 \mu \mathrm{g} / \mathrm{mL})$ HCCLM3 cells with or without the pretreatment of knockdown or overexpression via shRNA or pcDNA/peGFP vectors. $d$ Genes in central carbon metabolism pathway of YAP high expression group on KEGG graph rendered by pathview. Gene expression levels are indicated as significantly higher (red), unchanged (gray), or lower (green). e Barchart visualized significantly related genes in central carbon metabolism with higher YAP1 expression including 10 genes with the highest logFC and 4 genes with the lowest logFC from datasets GSE97098, GSE77314 and GSE153783. $f$ Western blotting analysis of the YAP and GLUT1 protein level of HCCLM3 cells treated with WZ35 and plasmid. g Predicted TEAD binding motif site sequence in the promoter region of GLUT1 host gene chromosome 1 from the database JASPAR. 
A

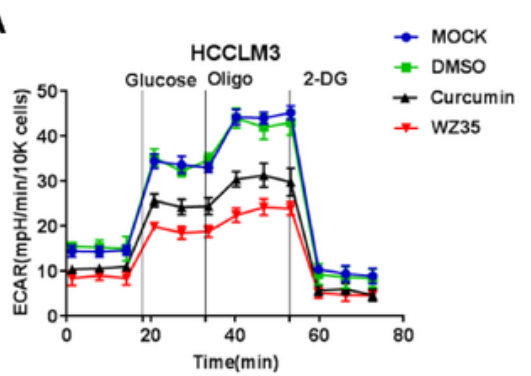

D

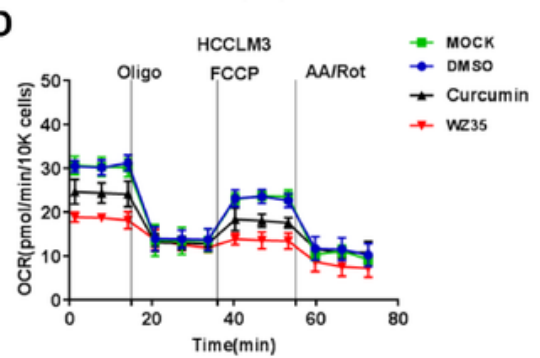

H

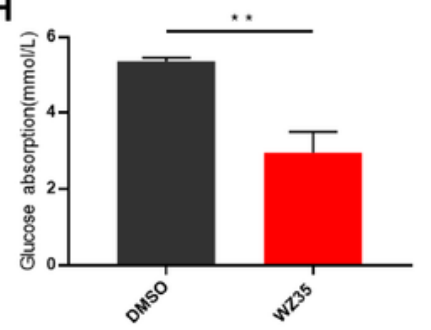

I
B

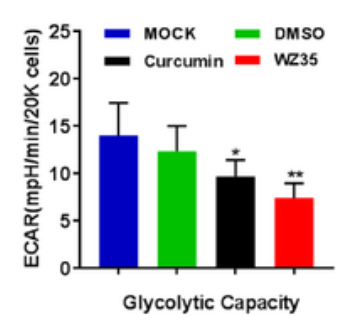

C

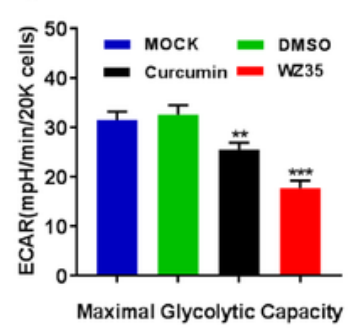

E
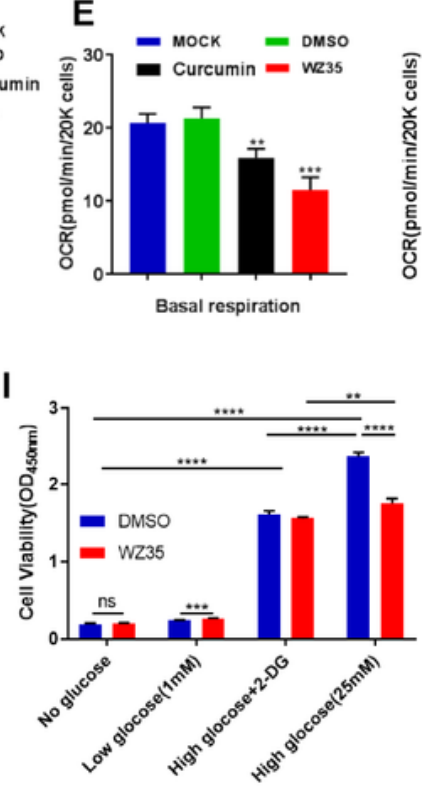

$\mathbf{K}$

Glucose intake

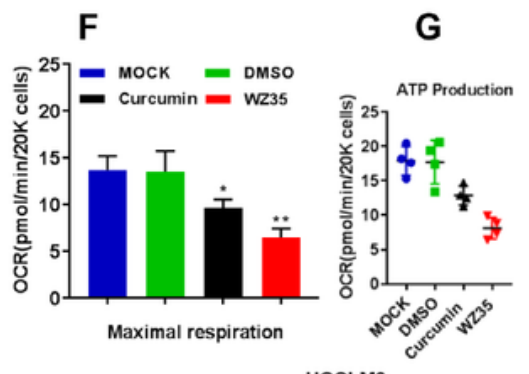

HCCLM3

J

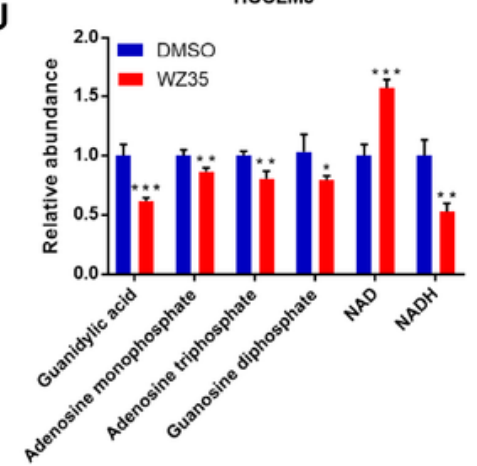

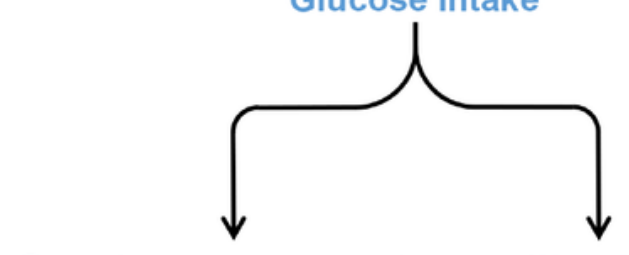

Oxidative phosphorylation Glycolysis

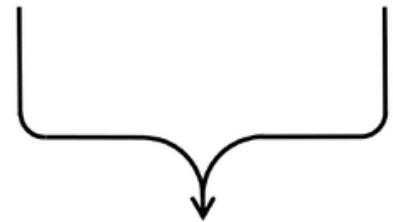

Purine metabolism

$\downarrow$

Proliferation

\section{Figure 6}

WZ35 caused a significant disturbance in the cellular metabolic state a,d ECAR and OCR was measured with Seahorse XF96 Flux analyzer in HCCLM3 cells. b,c Glycolytic capacity and maximal glycolytic capacity reserve were measured in non-glucose medium after the addition of glucose (10 mM), oligomycin $(1 \mu \mathrm{M})$ and 2-DG $(100 \mathrm{mM})$. e,f,g Basal respiration, maximal respiration and ATP production were measured after the injection of oligomycin $(1 \mu \mathrm{M})$, FCCP $(0.5 \mu \mathrm{M})$ and AA/Rot $(2 \mu \mathrm{M})$. h The glucose 
concentration of culture medium was detected by hexokinase method. i The absorption of glucose was measured in combination with CCK-8 assay in either non glucose-treated or low glucose-treated or high glucose-treated or high glucose plus 2-DG-treated HCCLM3 cells with or without treatment of WZ35. j The contents of metabolic products in HCCLM3 cells treated with or without WZ35 $(10 \mu \mathrm{g} / \mathrm{mL})$. All these results have been presented as the mean \pm standard error from independent experiments in triplicate. ${ }^{*} \mathrm{P}<$ $0.05,{ }^{*} \mathrm{P}<0.01,{ }^{\star * *} \mathrm{P}<0.001,{ }^{\star \star * *} \mathrm{P}<0.0001$, student's $\mathrm{t}$ test. $\mathrm{k}$ Proposed working model showed distinct metabolic changes after drug action.

A

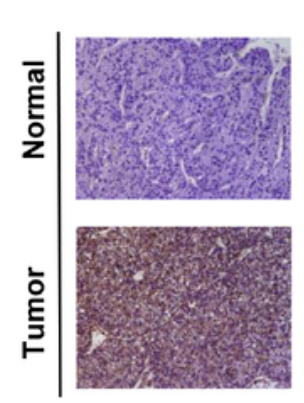

B

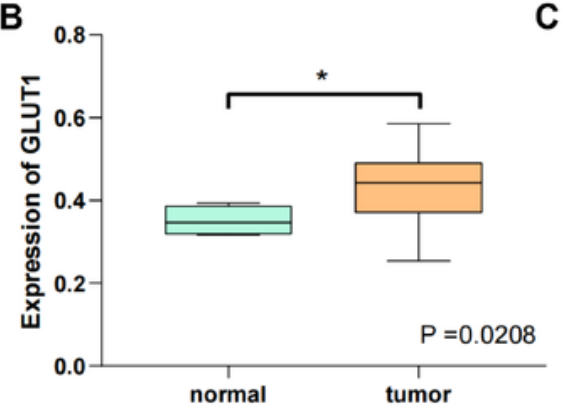

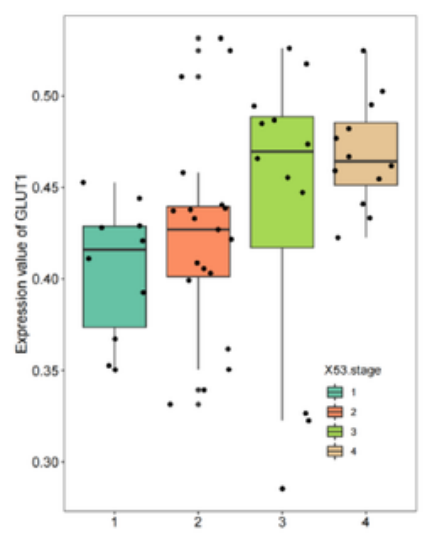

D
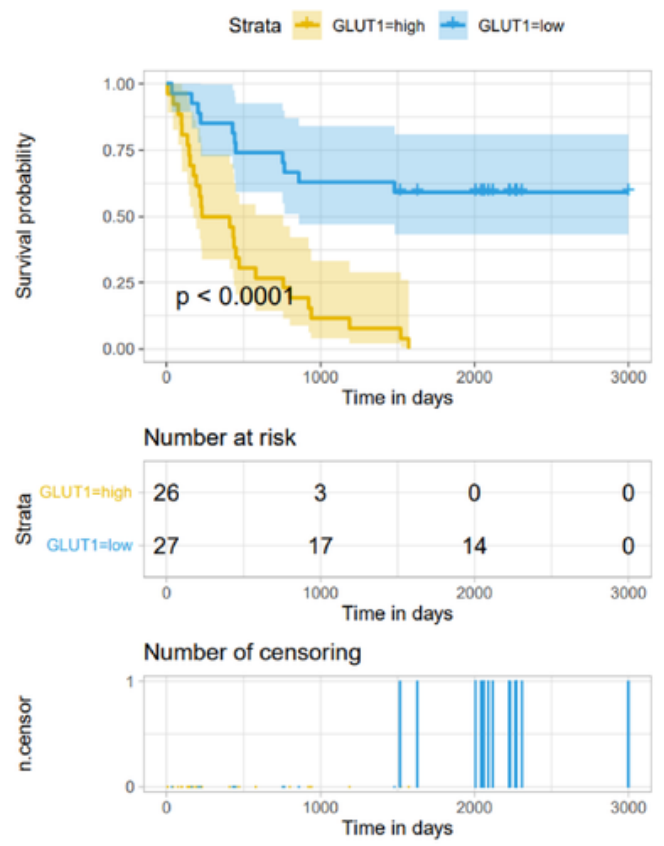

F

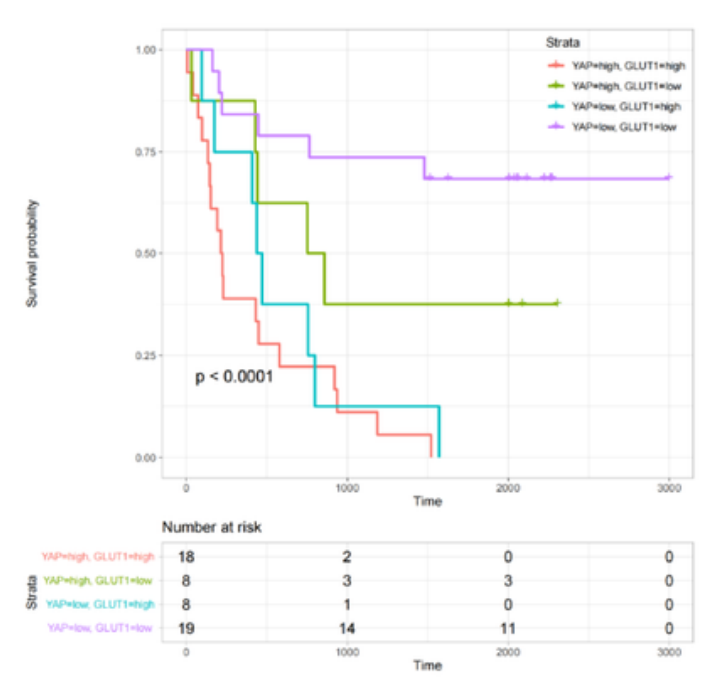

E

G

H
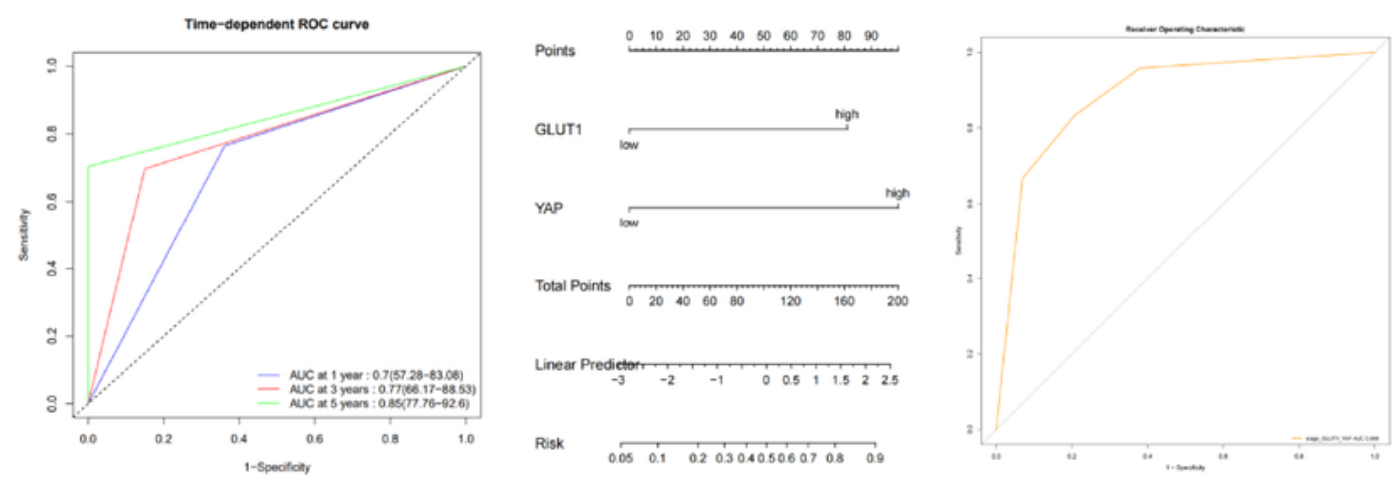


\section{Figure 7}

YAP combined GLUT1 may serve as a valuable prognostic and/or diagnostic biomarker in HCC a,b GLUT1 IHC staining in HCC patient samples. Images of cells were used to evaluate GLUT1 expression between the normal and the tumor. c GLUT1 IHC staining in HCC patient samples. Representative GLUT1stained images of TNM stage I, II, III and IV HCC tumor tissues were quantified. d Kaplan-Meier approach visualized the relationship between GLUT1 expression and OS in HCC patients ( $n=53, P<0.001)$ e Timedependent ROC curves analyzing the potential value of expression levels of GLUT1. $f$ Kaplan-Meier curve for the disease free survival and overall survival have been illustrated for the subgroups YAP high/GLUT1 low, YAP high/GLUT1 high, YAP low/GLUT1 low and YAP low/GLUT1 high. g Nomogram for predicting the stage according to the expression levels of GLUT1 and YAP. Instruction: Each characteristic was located on the corresponding variable axis, and a vertical line was drawn upwards to the points axis to determine the specific point value. This process was repeated and the total points value was tallied and located on the linear pretector axis. A vertical line was drawn down to the rank to obtain the probability of later stage $\mathrm{h}$ ROC calibration plots of the nomogram for combined YAP and GLUT1 1 The AUC was 0.889.

\section{Supplementary Files}

This is a list of supplementary files associated with this preprint. Click to download.

- SupplementryFigure.pdf 